\title{
Kommunale Kassenkredite: Bedeutung, Verursachung und Lösungsoptionen
}

\author{
von Martin Junkernheinrich und Uwe Wagschal
}

Die Verschuldungssituation der kommunalen Ebene Deutschlands, insbesondere die Verschuldung durch Kassenkredite stand in den letzten zehn Jahren verstärkt in der öffentlichen Diskussion. Im vorliegenden Beitrag wird die prekäre Situation mit zentralen Kennzahlen verdeutlicht. Ursachen der Neuverschuldung durch Kassenkredite werden mit Hilfe verschiedener ökonometrischer Methoden untersucht. Dabei stehen politische und sozioökonomische Indikatoren im Vordergrund. Ferner sollen unter anderem mit Hilfe einer Bürgermeisterbefragung aus Sicht von Kommunen, Ländern und Bund Handlungsmöglichkeiten zur Lösung der kommunalen Schuldenproblematik aufgezeigt werden. Im Ergebnis sind die Ausgaben - und Einnahmenpolitik der Kommunen, kommunale Entschuldungsprogramme der Länder und die Ausgabenentlastung im Sozialbereich von zentraler Bedeutung.

The debt situation at Germany's municipal level in particular short term debt has been a central public discussion point in the past decade. The in hand contribution shows the precarious situation with the help of key data. Determinants of new indebtedness are explored by means of econometric methods. Therefore, political and socio-economic indicators are of central interest. Furthermore, and also by the help of the survey among local mayors different approaches to write off debts are illustrated. This is done by highlighting the different points of view of the municipalities, of the federal states and also of the Bund. As a result, the municipal expenditure and income policy, as well as municipal debt relief programs by the federal states and the pressure because of social security are of central importance.

\section{Einleitung}

Die kommunale Verschuldung hat sich, wie die gesamte öffentliche Verschuldung, in den letzten Jahren - auf den ersten Blick - vergleichsweise gut entwickelt. Im Saldo ist die öffentliche Gesamtverschuldung der Kommunen zurückgegangen, es konnten sogar Überschüsse erwirtschaftet werden. Auf der anderen Seite verweisen jedoch einige Studien auf die hohe Schuldenlast in vielen Kom-

1 David Boll und Dominik Frankenberg, TU Kaiserslautern, sei an dieser Stelle für die Mitarbeit gedankt. 
munen und einen hohen Investitionsstau ${ }^{2}$. Zwischen den Kommunen in den einzelnen Bundesländern aber auch innerhalb einzelner Bundesländer können große Unterschiede ausgemacht werden. So weisen rund $35 \%$ der Kommunen in Deutschland eine moderate Verschuldung unter 500 Euro je Einwohner auf, viele davon sind sogar schuldenfrei. In einigen hoch verschuldeten Städten ist dagegen eine Kassenkreditverschuldung von knapp 7.000 Euro je Einwohner festzustellen, während die Gesamtverschuldung bis zu 12.000 Euro je Einwohner beträgt.

Die Kommunalverschuldung wurde in der Vergangenheit zumeist im Aggregat betrachtet, eine detaillierte Analyse einzelner Komponenten der Kommunalverschuldung unterblieb zumeist. ${ }^{3}$ Eine Besonderheit der Kommunalverschuldung in der Bundesrepublik Deutschland sind dabei die Kassenkredite (auch als Liquiditätskredite bzw. Kassenverstärkungskredite bezeichnet), die als kurzfristiger Ausgleich der Verwaltungstätigkeit der Kommunen dienen sollen. Diese waren erst in einer größeren Studie Gegenstand der Untersuchung. ${ }^{4}$ Allerdings untersuchten Heinemann et al. nur haushaltsspezifische (z.B. Ausgaben), sozioökonomische (Arbeitslosenquote, Gemeindegröße) und demographische Variablen (Altenquotient) und berücksichtigten nicht politische oder institutionelle Erklärungsgrößen der Verschuldung, denen in der politik-ökonomischen Fachwissenschaft eine Erklärungskraft zugeschrieben wird. Diese Faktoren wie etwa die parteipolitische Zusammensetzung des Kommunalparlaments, die Parteizugehörigkeit des Bürgermeisters, die Größe und die politische Zersplitterung des Kommunalparlaments, aber auch die Kommunalverfassung und die institutionalisierte Stärke des Bürgermeisters sollen im Mittelpunkt dieses Beitrages stehen.

Die Daten des Statistischen Bundesamtes zeigen, dass gerade die Kassenkredite in der jüngeren Vergangenheit dramatisch angestiegen sind. Zudem sind diese Kassenkredite in hohen Maße ein Phänomen in den westdeutschen Flächenländern (mit Ausnahme Baden-Württembergs und Bayerns). ${ }^{5}$ Die Kassenkredite, ursprünglich gedacht als kurzfristiger Liquiditätsausgleich der kommunalen

2 Vgl. Rehm, H./ Tholen, M.: Kommunalverschuldung - Befund, Probleme, Perspektiven, Berlin, 2008, hier 259.; Reidenbach, M. et al.: Investitionsrückstand und Investitionsbedarf der Kommunen. Ausmaß, Ursachen, Folgen, Strategien. Deutsches Institut für Urbanistik - Edition Stadt Forschung Praxis, Bd. 4. Berlin, 2008. hier $18 \mathrm{ff}$.

3 Eine integrierte Schuldenberichterstattung erfolgte erstmals mit dem „Schuldenreport“ der Bertelsmann Stiftung. Vgl. Junkernheinrich, M./Micosatt, G.: Kommunaler Finanz- und Schuldenreport Deutschland. Ein Ländervergleich, Bertelsmann Stiftung, Gütersloh, 2008.

4 Vgl. Heinemann, $F$. et al: Der kommunale Kassenkredit zwischen Liquiditätssicherung und Missbrauchsgefahr, Baden-Baden, 2009.

5 Vgl. Eltges, M./Müller-Kleissler, R.: Kommunale Kassenkredite. Hausgemacht oder strukturell begründbar? Expertise im Auftrag des Bundesministeriums der Finanzen. Bonn, 2010. 
Haushalte, stehen somit im Mittelpunkt dieses Beitrages, da deren außergewöhnliche Varianz zwischen den Bundesländern erklärungsbedürftig sind. Mit dem Fokus auf den Kassenkreditbestand als abhängige Variable ist gegenüber der Haushaltsergebnisorientierung der Vorteil verbunden, das kumulative Ergebnis der Vergangenheit vollständig abzubilden, da ungelöste Deckungsprobleme umfassender erfasst sind und hier in der Regel kein striktes Ausgleichsziel vorliegt. Aus finanzwissenschaftlicher Sicht sind - längerfristig aufgenommene Kassenkredite besonders problematisch, weil damit strukturelle Haushaltslücken (auch Personal- und Sozialausgaben) finanziert werden, denen im Gegensatz zu Investitionskrediten kein Vermögensaufbau gegenübersteht. Damit besteht die Gefahr einer kommunalen Schuldenfalle, bei der immer größere Anteile des Haushaltes durch Zinszahlungen belastet werden und die Tilgung immer schwieriger wird.

Dazu werden in Abschnitt 2 zunächst die Verteilung, die Höhe und die relative Bedeutung der kommunalen Kassenkredite diskutiert. Daran anschließend werden in Abschnitt 3 die Determinanten der Kassenkredite näher untersucht; insbesondere wird dabei auf die sozioökonomischen, die institutionellen und die politischen Bestimmungsfaktoren der Höhe der Kassenkredite eingegangen. Schließlich werden im Abschnitt 4 zentrale Maßnahmen zum Abbau der hohen Kassenkreditbestände diskutiert. Diese können einerseits auf eigene Maßnahmen der Kommunen abzielen, aber auch auf Hilfsleistungen der Bundesländer oder Bundes. Abschnitt 5 fasst die wichtigsten Ergebnisse zusammen.

\section{Kassenkredite als Teil der Kommunalverschuldung}

Gemessen an dem Schuldenstand je Einwohner scheint die kommunale Verschuldung bezogen auf dem öffentlichen Gesamthaushalt (Bund, Länder, Sondervermögen des Bundes sowie Gemeinden und Zweckverbände) nicht sonderlich dramatisch zu sein, denn nur rund $7 \%$ der Gesamtverschuldung entfällt auf die Kommunen (1.807 Euro je Einwohner). Diese liegt Ende 2013 in Deutschland bei 25.289 Euro je Einwohner. Davon entfallen weiter etwa $62 \%$ auf den Bund (15.860 Euro je Einwohner), rund $31 \&$ auf die Länder (7.755 Euro je Einwohner) und ein marginaler Teil auf die Sozialversicherungen (10 Euro je Einwohner).

Betrachtet man die Verschuldungsentwicklung, so liegt die Pro-KopfVerschuldung der Kommunen 2013 um 87\% über dem Wert von 1991 (1.807 Euro zu 966 Euro). Rechnet man die Inflation aus dem Verschuldungsanstieg heraus, dann ist die Verschuldung 2013 im Vergleich zum Basisjahr 1991 
preisbereinigt um rund $27 \%$ gestiegen, d.h. jahresdurchschnittlich um knapp 1,2 Prozent. Dagegen hat der Bund seine Verschuldung im selben Zeitraum um rund $360 \%$ erhöht, also mehr als viermal so viel.

Im Verhältnis zum Bruttoinlandsprodukt (BIP) ist die Entwicklung der Verschuldung der kommunalen Kernhaushalte im Wesentlichen identisch zur ProKopf-Entwicklung. Insgesamt fällt auf, dass für den Gesamthaushalt bereits seit 2003 durchgehend das Schuldenstandskriterium des Vertrages von Maastricht verletzt wird. Der Bund trägt dabei den Hauptanteil der Verschuldung. Die Kommunalverschuldung ist - gemessen in Prozent des BIP - nahezu konstant geblieben (1991: 4,7\%; 2012: 5,1 \% des BIP). Dieser Sachverhalt ist jedoch hauptsächlich ein Ergebnis der Nivellierungswirkung des hohen Aggregationsniveaus. Dagegen hat sich die Verschuldung der Länder von 11,7 \% des BIP auf $24,4 \%$ des BIP mehr als verdoppelt.

Ein zunehmend wichtiger Bestandteil der Schuldenanalyse ist die Schuldenstruktur. Auf der Ebene der Kommunen kann man beobachten, dass das Instrument der Kassenkredite deutlich zugenommen hat. ${ }^{6}$ Unter Kassenkrediten werden die kurzfristigen Verbindlichkeiten erfasst, die die Berichtseinheiten zur Überbrückung vorübergehender Kassenanspannungen eingehen. Sie dienen nicht der investiven Ausgabendeckung, sondern der Aufrechterhaltung einer ordnungsgemäßen Kassenwirtschaft beziehungsweise der Liquiditätssicherung und damit der Finanzierung laufender Ausgaben. Ende 2013 liegt der Anteil der Kassenkredite an der gesamten Kommunalverschuldung im Saarland bei über $62 \%$, während er in Rheinland-Pfalz und in Nordrhein-Westfalen bei etwa $50 \%$ liegt. Eine Gruppe mit einem mittelgroßen Anteil an der Verschuldung besteht aus den drei westdeutschen Bundesländern Hessen, Niedersachsen und SchleswigHolstein sowie den ostdeutschen Bundesländern (vgl. Tab. 1). Ein detaillierter Blick auf die Verschuldungsdaten zeigt, dass 94,1 \% aller Kassenkredite auf die westdeutschen Bundesländer entfallen. Und in den westdeutschen Bundesländern macht der Anteil der Kassenkredite an der Gesamtheit der Verschuldung der Kernhaushalte $33 \%$ aus.

6 Vgl. Boettcher, F.: Ursachen kommunaler Haushaltsdefizite: Eine finanz- und politikwissenschaftliche Untersuchung am Beispiel der nordrhein-westfälischen Gemeinden, Münster, 2013. 
Tabelle 1: Kommunale Verschuldungsindikatoren Pro-Kopf-Verschuldung in den Bundesländern (inkl. Extrahaushalte, Zweckverbände, FEU)

\begin{tabular}{|c|c|c|c|c|}
\hline Bundesland ${ }^{1}$ & $\begin{array}{l}\text { Durchschnitt- } \\
\text { liche kommu- } \\
\text { nale Pro-Kopf- } \\
\text { Verschuldung } \\
\text { in Euro (inkl. } \\
\text { Extrahaushal- } \\
\text { te, Zweckver- } \\
\text { bände, FEU) } \\
\text { am } 31.12 .12^{2} \\
\end{array}$ & $\begin{array}{l}\text { Durchschnitt- } \\
\text { liche kommu- } \\
\text { nale Pro-Kopf- } \\
\text { Verschuldung } \\
\text { in Euro in den } \\
\text { Kern- und } \\
\text { Extrahaushal- } \\
\text { ten (31.12.13) }\end{array}$ & $\begin{array}{l}\text { Kassenkredite } \\
\text { in Euro und } \\
\text { Pro-Kopf der } \\
\text { Kern- und } \\
\text { Extrahaushal- } \\
\text { ten }(31.12 .13)\end{array}$ & $\begin{array}{l}\text { Anteil der } \\
\text { Kassenkredite } \\
\text { an der Ver- } \\
\text { schuldung des } \\
\text { Kernhaushal- } \\
\text { tes }\end{array}$ \\
\hline $\begin{array}{l}\text { Schleswig- } \\
\text { Holstein }\end{array}$ & 2.175 & 1.442 & 271 & 18,8 \\
\hline Bayern & 2.293 & 1.117 & 21 & 1,9 \\
\hline Sachsen & 2.753 & 1.068 & 26 & 2,5 \\
\hline $\begin{array}{l}\text { Baden- } \\
\text { Württemberg }\end{array}$ & 2.856 & 632 & 15 & 2,4 \\
\hline Thüringen & 2.901 & 1.425 & 94 & 6,6 \\
\hline Niedersachsen & 2.921 & 1.589 & 474 & 29,8 \\
\hline Brandenburg & 3.050 & 868 & 322 & 37,1 \\
\hline Sachsen-Anhalt & 3.678 & 1.394 & 467 & 33,5 \\
\hline $\begin{array}{l}\text { Mecklenburg- } \\
\text { Vorpommern }\end{array}$ & 4.013 & 1.195 & 400 & 33,4 \\
\hline Rheinland-Pfalz & 4.233 & 2.977 & 1.496 & 50,2 \\
\hline $\begin{array}{l}\text { Nordrhein- } \\
\text { Westfalen }\end{array}$ & 4.426 & 2.859 & 1.439 & 50,3 \\
\hline Hessen & 5.173 & 3.010 & 1.091 & 36,3 \\
\hline Saarland & 6.220 & 3.268 & 1.957 & 59,9 \\
\hline Durchschnitt $^{3}$ & 3.468 & 1.807 & 634 & 35,1 \\
\hline
\end{tabular}

Anmerkungen: Die Tabelle ist nach der Höhe der gesamten Kommunalverschuldung sortiert. 1 = Die Stadtstaaten Bremen, Berlin und Hamburg werden in den amtlichen Finanzstatistiken nicht der kommunalen, sondern der staatlichen Ebene zugeordnet. $2=$ Schulden je Einwohner als Summe aus Wertpapierschulden, Kassenkrediten und Krediten beim nicht-öffentlichen Bereich am 31. Dezember 2012, bezogen auf die Einwohnerzahl (auf Basis des Zensus 2011) zum 30. Juni 2012. 3 = Schulden des öffentlichen Bereichs (ohne private Anteile) am 31. Dezember 2012, bezogen auf die Einwohnerzahl des Bundeslandes. 3 = gewichtet mit der Bevölkerung in den einzelnen Bundesländern. FEU = öffentlichen Fonds, Einrichtungen und Unternehmen. 
Außerdem ist die Verschuldung auf kommunaler Ebene weitaus problematischer als es die Daten für die Kernhaushalte suggerieren. Dies liegt an den Auslagerungen in Extrahaushalten sowie den Schulden von öffentlichen Fonds, Einrichtungen und Unternehmen (FEU), die in unterschiedlichsten Rechtsformen (z.B. Eigenbetriebe, Zweckverbände) und Beteiligungsquoten geführt werden. Die Finanzstatistik hat diese Verschuldung bisher nicht systematisch ausgewiesen. Mit dem Aufbau eines konsolidierten Berichtssystems, dem „Schalenkonzept der öffentlichen Aufgabenwahrnehmung“" wurde es möglich diese zusätzliche kommunale Verschuldung zu erfassen. Das Statistische Bundesamt hat diese integrierten kommunalen Schulden nach dem Schalenkonzept erstmalig 2014 veröffentlicht. ${ }^{7}$ Dabei werden neben den Schulden der kommunalen Kernhaushalte auch die Schulden der Extrahaushalte und sonstigen öffentlichen Fonds, Einrichtungen und Unternehmen, an denen die Kommunen unmittelbar oder mittelbar beteiligt sind, miteinbezogen und anteilig entsprechend den jeweiligen Beteiligungsverhältnissen zugerechnet. Die Daten in Spalte 1 in Tabelle 1 zeigen dabei - im Aggregat - ein deutlich höheres Verschuldungsniveau der kommunalen Ebene. Die Pro-Kopf-Verschuldung steigt im Bundesdurchschnitt von 1.810 Euro (Stand: 1.807 Euro zum Zeitpunkt 31.12.2013) um knapp das Doppelte auf 3.468 Euro. An der Spitze des kommunalen Verschuldungsrankings liegen bei dieser weiter gefassten Betrachtung Rheinland-Pfalz, NordrheinWestfalen, Hessen und das Saarland, während eine niedrige kommunale Verschuldung in Schleswig-Holstein, Bayern, Sachsen und Baden-Württemberg zu beobachten ist.

Legt man zudem eine Grenze von 2.000 Euro an diesem weitgefassten Indikator an, kann man damit den Anteil der hochverschuldeten Kommunen ausweisen. Wiederum ist eine starke regionale Varianz zwischen den Bundesländern, aber auch innerhalb der einzelnen Bundesländer feststellen. So sind im Saarland alle 52 Kommunen hoch verschuldet. In Rheinland-Pfalz gibt es zwar eine sehr hohe Durchschnittsverschuldung, aber es gibt nur wenige (2,6 \% aller Kommunen und damit den aufgrund der kleinteiligen Gemeindestruktur niedrigsten Anteil unter den Bundesländern) hochverschuldete Kommunen. Dabei sind gerade in diesen Kommunen die Kassenkredite hoch und konzentrieren sich auf die wenigen kreisfreien Städte. ${ }^{8}$

7 Vgl. Statistisches Bundesamt: Integrierte Schulden der Gemeinden und Gemeindeverbände, Wiesbaden, 2014.

8 Vgl. Junkernheinrich, M./Micosatt, G., a.a.O.; Holtkamp, L.: Kommunale Haushaltspolitik bei leeren Kassen. Bestandsaufnahme, Konsolidierungsstrategien, Handlungsoptionen; 2. Auflage, Berlin, 2012. 
Die Relevanz der Kassenkredite als Untersuchungsgegenstand zeigt sich auch daran, dass sich diese in 15 Jahren (bis zum Jahr 2013) auf insgesamt 47,4 Milliarden Euro und damit fast verneunfacht haben. ${ }^{9}$ Der Kassenkredit wird von einem kurzfristigen zu einem permanenten Verschuldungsinstrument, dessen Bedeutung massiv zunimmt.

Abbildung 1: Die 25 Kommunen mit den höchsten Kassenkrediten (2012)

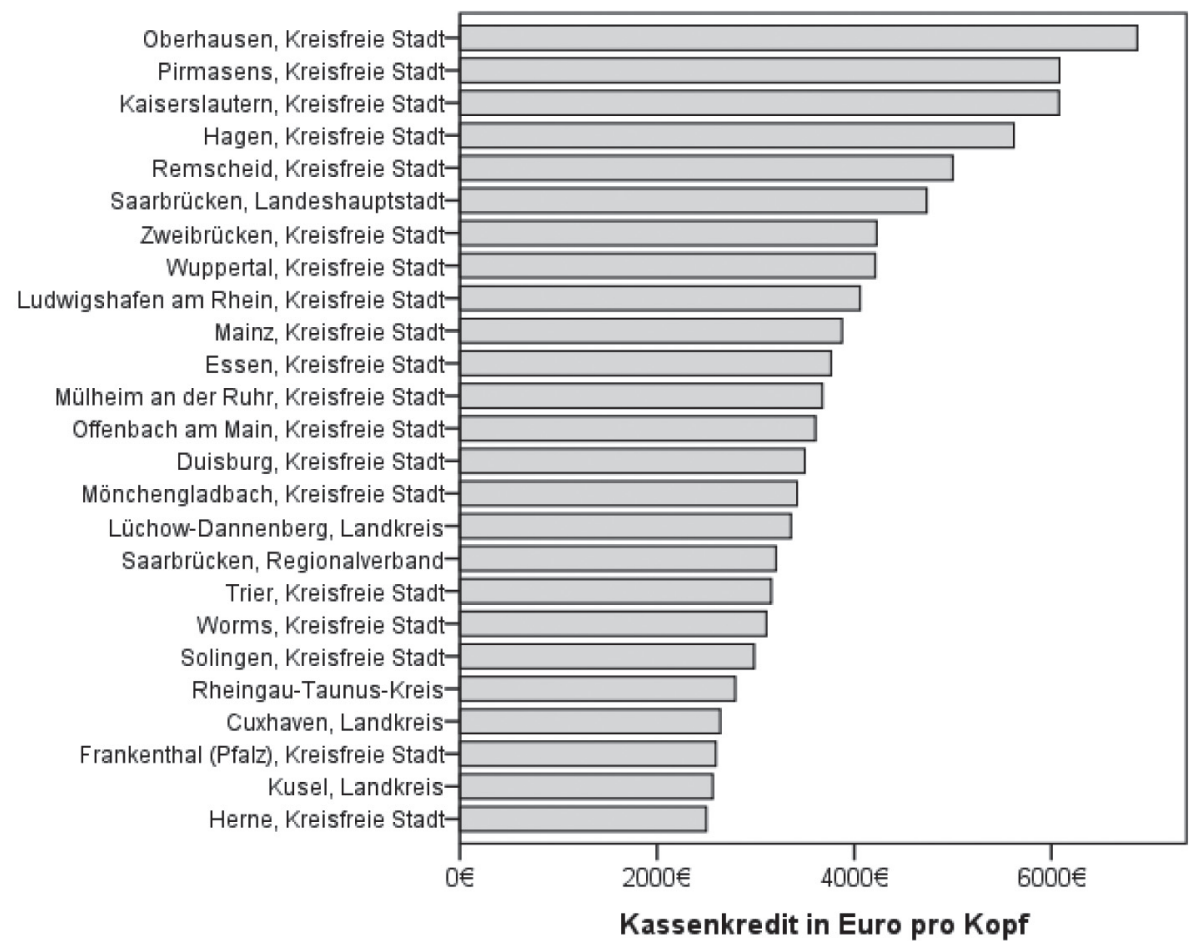

Quelle: Regionaldatenbank des Statistischen Bundesamtes

Eine detaillierte Betrachtung der 25 Städte mit den höchsten Kassenkrediten im Jahr 2012 (vgl. Abb. 2) zeigt, dass die meisten Kommunen mit hohen Kassenkrediten zwar aus Nordrhein-Westfalen (10), Rheinland-Pfalz (8), dem Saarland (3), Hessen und Niedersachsen (je 1) sind. Aber auch innerhalb der hoch belasteten Länder gibt es zahlreiche Kreise und Städte, die mit einer geringen Verschuldung aufwarten können. So haben 16 der 53 Kreise in NordrheinWestfalen weniger als 300 Euro Kassenkredite und der Median liegen bei

9 Insgesamt haben sich die Kassenkredite seit 1992, also in 20 Jahren, sogar mehr als verdreißigfacht. 
799 Euro, d.h. die Hälfte der Kreise hat Kassenkredite unterhalb dieser Grenze. Insbesondere sind es westdeutsche strukturschwache Städte und Kreise, die einem starken sozioökonomischen Strukturwandel unterliegen und u.a. daher eine hohe Verschuldung aufweisen.

\section{Determinanten der Kassenkredite}

\section{Theoretische Überlegungen und Hypothesen}

Welche Faktoren sind verantwortlich für die Höhe der Kassenkredite. In der Literatur ${ }^{10}$ wurden in der Vergangenheit vor allem exogene Variablen dafür verantwortlich gemacht, wie die Arbeitslosenquote, der Anteil älterer Menschen an der Bevölkerung (Altenquotient), der Anteil der Sozialhilfeempfänger, aber auch die Gemeindegröße. Sozioökonomische Probleme bündeln sich - so die Hypothese - besonders in Großstädten, die zudem häufig einem stärkeren Strukturwandel der industriellen Beschäftigung unterliegen.

Die Varianz zwischen den Gemeindegrößenklassen ist beachtlich. Der in Tabelle 2 dargestellte Mittelwertvergleich zwischen den vier verschuldungsrelevanten Größen auf kommunaler Ebene:

(1) Primärsaldo des Verwaltungshaushalts = laufende Einnahmen - laufende Ausgaben (einschl. Investitionen), ${ }^{11}$

(2) Ordentliches Ergebnis des Kernhaushalts = Primärsaldo + Finanzsaldo,

(3) Gesamtverschuldung = Schuldenstand an Krediten, Wertpapierkrediten und Kassenkrediten sowie

(4) Bestand an Kassenkrediten

zeigt eine überdurchschnittliche Verschuldung - sowohl bei den beiden jährlichen Stromgrößen als auch den beiden Bestandsgrößen - bei den Städten im Bereich von 50.000 bis 500.000 Einwohnern. Die Kassenkreditbelastung ist mit durchschnittlich über 1.500 Euro je Einwohner in der Gebietskörperschaften der Größenklasse von 100.000 bis 200.000 Einwohner am höchsten.

10 Vgl. Heinemann et al., a.a.O.

11 Diese Abgrenzung ist von einer anderen Verwendung des Begriffs Primärsaldos zu unterscheiden. Bei dieser Größe werden die Zinszahlungen vom Bruttohaushaltssaldo subtrahiert und die Vermögenserlöse hinzugerechnet (Vgl. Wagschal, U. / Wenzelburger, G.: Haushaltskonsolidierung, Wiesbaden, 2008). 
Tabelle 2: Verschuldungsgrößen und Gemeindegrößenklassen (2010)

\begin{tabular}{|c|c|c|c|c|}
\hline Bevölkerungsklassen & Primärsaldo & $\begin{array}{l}\text { Ordentliches } \\
\text { Ergebnis } \\
\text { Kernhaushalt }\end{array}$ & $\begin{array}{l}\text { Gesamt- } \\
\text { verschuldung }\end{array}$ & Kassenkredite \\
\hline 5000 bis unter 10.000 & -93 & -111 & 1.064 & 97 \\
\hline 10.000 bis unter 20.000 & -95 & -109 & 1.529 & 188 \\
\hline 20.000 bis unter 50.000 & -103 & -115 & 1.979 & 279 \\
\hline 50.000 bis unter 100.000 & -133 & -173 & 3.218 & 653 \\
\hline 100.000 bis unter 200.000 & -141 & -182 & 5.199 & 1.511 \\
\hline 200.000 bis unter 500.000 & -156 & -184 & 5.144 & 1.282 \\
\hline Über 500.000 & -91 & -48 & 2.361 & 267 \\
\hline Gesamtdurchschnitt & -110 & -122 & 2.658 & 473 \\
\hline
\end{tabular}

Anmerkungen: Eigene Auswertung. Dargestellt sind die Mittelwerte für die vier Verschuldungsindikatoren (in Euro pro Kopf)

Die Großstädte über 500.000 Einwohner schneiden dagegen etwas besser ab als die Mittelstädte und kleinere Großstädte. Die finanzielle Situation für die Kommunen in Deutschland kann damit nicht einheitlich beurteilt werden, sondern ist immer kontextabhängig und hängt beispielsweise von der Raumstruktur (z.B. der Eigenheimquote), der Kaufkraft und auch dem Anteil der Sozialhilfeempfänger (SBG II-Quote) und der Arbeitslosenquote ab.

Zur Erklärung der Verschuldungsursachen werden in der Literatur sowohl exogene als auch endogene Faktoren diskutiert. ${ }^{12}$ Exogene Faktoren sind kommunal nur eingeschränkt beeinflussbare Faktoren, wie etwa lokale sozioökonomische Problemlagen (z.B. Arbeitslosenquote). Dabei können aber auch Institutionen exogen sein, wie etwa die unterschiedlichen Ordnungsrahmen auf den einzelnen staatlichen Ebenen. Ein wichtiger Faktor ist zudem die Kommunalaufsicht, die für die Genehmigung von Liquiditätskrediten zuständig ist. In der Praxis sind Kassenkredite allerdings nicht in allen Bundesländern genehmigungsbedürftig, sondern unterliegen lediglich einer Anzeige- bzw. Vorlagepflicht.

12 Vgl. Timm-Arnold, K.-P.: Bürgermeister und Parteien in der kommunalen Haushaltspolitik, Wiesbaden, 2010.; Holtkamp, L.; a.a.O.; Boettcher, F. a.a.O.; Wagschal, U.: Staatsverschuldung. Ursachen im internationalen Vergleich, Opladen, 1996.; Junkernheinrich, M. et al:: Haushaltsausgleich und Schuldenabbau - Konzept zur Rückgewinnung kommunaler Finanzautonomie im Land NordrheinWestfalen, Berlin, 2014. 
Endogene Faktoren wirken vor allem gemeindespezifisch und sind vom politischen Wettbewerb vor Ort determiniert. Insbesondere der Parteienwettbewerb, die Reaktion auf anstehende Wahlen, die lokale politische Kultur, die entweder konsensdemokratisch oder wettbewerblich ausgerichtet sein kann, sind wichtige Faktoren. Auch die Stärke des Bürgermeisters kann, obwohl durch die Kommunalverfassung exogen fixiert, zu einer endogenen Größe werden, wenn das faktische Verhalten berücksichtigt wird. In Abbildung 2 werden exogene und endogene Faktoren systematisch dargestellt.

Abbildung 2: Zentrale Einflussfaktoren der kommunalen Finanzlage

\begin{tabular}{|c|c|c|}
\hline Einflussfaktoren & Gestaltbarkeit & räumliche Spezifität \\
\hline lokale sozioökonomische Position & \multirow{6}{*}{ gemeindeexogen } & gemeindespezifisch \\
\hline raumstrukturelle Prägung & & \multirow{5}{*}{ landeseinheitlich } \\
\hline konjunkturelle Lage & & \\
\hline finanzpolitischer Ordnungsrahmen & & \\
\hline $\begin{array}{l}\text { kommunalpolitischer Ordnungs- } \\
\text { rahmen }\end{array}$ & & \\
\hline $\begin{array}{l}\text { haushaltsrechtlicher Ordnungsrah- } \\
\text { men }\end{array}$ & & \\
\hline Kommunalaufsicht & gemeindeexogen & $\begin{array}{l}\text { gemeindespezifisch / } \\
\text { landeseinheitlich }\end{array}$ \\
\hline $\begin{array}{l}\text { kommunalpolitische Willensbil- } \\
\text { dung } \\
\text { (wettbewerblich oder konkordant) }\end{array}$ & gemeindeendogen & gemeindespezifisch \\
\hline Stellung des Bürgermeister & gemeindeendogen & gemeindespezifisch \\
\hline $\begin{array}{l}\text { Parteipolitische Kräfteverhältnisse } \\
\text { in der Kommune }\end{array}$ & gemeindeendogen & gemeindespezifisch \\
\hline
\end{tabular}

Quelle: Eigene Darstellung in Anlehnung an Boettcher 2013

Für den empirischen Test in den beiden nachfolgenden Abschnitten werden verschiedene Hypothesen getestet, die auf die Unterscheidung von exogenen und endogenen Faktoren zurückgeht. Dazu werden noch weitere in der Literatur verwendete Kontrollfaktoren verwendet, wie etwa die oben diskutierte Bevölkerungsgröße. Die zentralen Hypothesen sind: 
H1: Die sozioökonomischen Rahmenbedingungen beeinflussen die Kassenkreditentwicklung.

H1a: Ein höherer Bevölkerungsstand führt aufgrund der spezifischen Kosten der Ballung zu höheren Kassenkrediten.

H1b: Ein höherer Altenquotient der Bevölkerung führt aufgrund geringerer Erwerbstätigkeit und altersspezifischer Ausgabenbedarfe zu einem höheren Bestand an Kassenkrediten.

H1c: Eine höhere lokale Wirtschaftskraft (höhere Erwerbstätigenquote, Kaufkraft und Erwerbstätigenanteil im Dienstleistungssektor etc.) hat hingegen einen abschwächenden Effekt auf die Höhe der Kassenkredite.

H1d: Eine höhere Zentralität einer Kommune aufgrund einer höheren Bedeutung als Arbeitsort und Dienstleistungszentrum geht tendenziell mit Sonderlasten einher.

H2: Je schlechter die Finanzausstattung einer Kommune desto höher sind die Kassenkredite.

H3a: Je höher der Anteil der Beschäftigten einer Gemeinde (bzw. je höher die Personalausgaben H3b) desto höher sind die Kassenkredite einer Kommune.

H4a:Je stärker Linksparteien im Gemeinderat/Kommunalparlament vertreten sind, desto höher sind die Kassenkredite. Für die Stärke der bürgerlichen Parteien gilt die gegenteilige Vermutung (Hypothese 4b).

H5a: Gehört der Bürgermeister einer Linkspartei (SPD, Linke oder Grüne) an, desto höher sind die Kassenkredite. Für die CDU/CSU-Bürgermeister gilt die gegenteilige Vermutung (Hypothese 5b).

H6: Vor Wahlen verschulden sich Kommunen stärker.

H7: Je stärker die Machtstellung des Bürgermeisters, desto niedriger werden die Höhe der Kassenkredite einer Kommune sein. ${ }^{13}$

H8: Eher konsensdemokratische Kommunen haben niedrigere Kassenkredite als Kommunen die einen stark polarisierten Wettbewerb haben.

13 Dies wird über einen Index von Bogumil und Holtkamp gemessen, der die institutionelle Stärke des Bürgermeisters misst. Vgl. Bogumil, J./ Holtkamp, L.: Kommunalpolitik und Kommunalverwaltung Eine praxisorientierte Einführung, bpb-Schriftenreihe, Bonn, 2013. 


\section{Empirische Befunde von Querschnittsregressionen}

Die statistischen Analysen der zuvor formulierten Hypothesen erfolgen anhand zweier statistischer Verfahren und Untersuchungsdesigns. Zunächst einmal wird das Niveau der Kassenkredite in einer Querschnittsregression untersucht. Daran anschließend (Kapitel 3.3) werden die Veränderungen der Kassenkredite mittels einer gepoolten Zeitreihenanalyse untersucht. Untersucht werden dabei die Städte und Gemeinden Deutschlands mit mehr als 10.000 Einwohner.

Das Untersuchungsdesign zur Erklärung des Niveaus der Kassenkredite basiert auf einem Erklärungsansatz der Staatstätigkeitsschule („Heidelberger Schule“), der verschiedene Theorien und Erklärungsansätze der Staatstätigkeit verknüpft und die endogenen und exogenen Verschuldungsursachen (vgl. Tab. 2) in den Blick nimmt. Dabei werden zunächst in Gleichung 1 in Tabelle 3 Variablen der sozioökonomischen Schule (die Höhe der Ausgaben für Grundsicherung und die ökonomische Bedeutung als Arbeitsort) verwendet. Darüber hinaus wird mit dem Niveau der Gesamtverschuldung eine Variable, die die Pfadabhängigkeit misst, mitberücksichtigt. Etwas problematisch ist dabei, dass ein Teil der abhängigen Variablen, nämlich die Kassenkredite, in der Gesamtverschuldung mitgemessen wird. Darüber hinaus werden noch raumgeographische Faktoren berücksichtigt, nämlich, ob sich eine Kommune in Ost- oder Westdeutschland befindet und ob eine Gemeinde kreisfrei oder kreisangehörig ist. Dies hat für die Kommunalhaushalte durchaus wichtige Implikationen, denn kreisfreie Städte bündeln viele sozioökonomische Problemlagen. Überdies sind kreisangehörige Städte kleiner und die Sozialausgaben im Kreis werden über Kreisumlagen finanziert, sodass kreisfreie Städte - so die Vermutung - deutlich höhere Verschuldung haben sollten als kreisangehörige Städte. Darüber hinaus werden noch zwei wichtige Einnahmen- bzw. Ausgabenfaktoren betrachtet: (1) die Höhe des Gewerbesteueraufkommens als Maß für die ökonomische Stärke einer Kommune. Dabei lautet die Hypothese, dass ein hohes Gewerbesteueraufkommen eine niedrige Verschuldung zur Folge hat. (2) Die Höhe der Personalausgaben, wobei als Hypothese angenommen wird, dass je mehr Personalausgaben getätigt werden, desto höher wird die Verschuldung durch Kassenkredite sein.

Die Ergebnisse dieser Faktoren zeigen, dass in der Tat die sozioökonomischen Problemlagen sowie die raumstruktuellen Faktoren hoch signifikant und erklärungskräftig sind. Auch die Pfadabhängigkeitsvariable ist hoch signifikant. Die Erklärungskraft des Modells ist eher mittelstark. Jedoch zeigt sich, dass das Modell, gemessen an den Problemen der Multikollinearität und Ausreißer kein statistisches Problem aufweist. 
In einem zweiten Schritt werden diesem eher traditionellen (ökonomischen) Erklärungsmodell öffentlicher Finanzen Variablen hinzugefügt, die Institutionen operationalisieren. Dies sind (vgl. Gleichung 2) der Kommunalverfassungsindex von Bogumil und Holtkamp sowie die Größe des Gemeinderates und parteipolitische Fragmentierung. Es zeigt sich, dass insbesondere der Kommunalverfassungsindex, der die Stärke der Bürgermeister misst, einen deutlichen signifikanten Einfluss auf die Höhe der Kassenkredite hat. Je stärker die Stellung des Bürgermeisters, desto tiefer sind die Kassenkredite, d.h. Hypothese 7 kann nicht widerlegt werden. Dies kann einerseits mit der starken Stellung des Bürgermeisters im Haushaltsprozess der Länder mit einer starken Kommunalverfassung begründet werden, aber auch der starken Stellung des Bürgermeisters per se, der seine Wiederwahl eng mit dem Zustand der öffentlichen Finanzen verknüpft sieht.

Von den soziökonomischen Faktoren können aufgrund der Multikollinearität nicht alle im Datensatz erfassten Faktoren geprüft werden, da diese insbesondere mit dem Anteil der Empfänger der Grundsicherung hoch korrelieren. Hierzu zählt etwa der Anteil der Arbeitslosen in einer Kommune oder auch die Absorptionsquote, d.h. der Anteil der Sozialausgaben in den Kommunen. Generell kann man jedoch feststellen, dass es gerade soziökonomische Problemlagen sind, die die Verschuldung besonders beeinflussen. 
Tabelle 3: Determinanten der Höhe der kommunalen Kassenkredite (2010)

\begin{tabular}{|c|c|c|c|c|c|}
\hline & (1) & (2) & (3) & (4) & (5) \\
\hline Gesamtverschuldung & $\begin{array}{r}0,11 * * * \\
(14,05)\end{array}$ & $\begin{array}{r}0,12 * * * \\
(11,44)\end{array}$ & $\begin{array}{r}0,11 * * * \\
(14,08)\end{array}$ & $\begin{array}{r}0,13^{* * *} \\
(11,90)\end{array}$ & $\begin{array}{r}0,12^{* * * *} \\
(11,87)\end{array}$ \\
\hline $\begin{array}{l}\text { Anteil der Empfänger } \\
\text { von Grundsicherung }\end{array}$ & $\begin{array}{r}38,96 * * * \\
(9,90)\end{array}$ & $\begin{array}{r}46,12 * * * \\
(7,04)\end{array}$ & $\begin{array}{r}39,46^{* * * *} \\
(10,09)\end{array}$ & $\begin{array}{r}48,77 * * * \\
(7,80)\end{array}$ & $\begin{array}{r}45,81^{* * * *} \\
(7,00)\end{array}$ \\
\hline $\begin{array}{l}\text { Bedeutung als Arbeits- } \\
\text { ort }\end{array}$ & $\begin{array}{r}-285,7^{* * * *} \\
(-6,88)\end{array}$ & $\begin{array}{r}-329,3 * * * \\
(-4,99)\end{array}$ & $\begin{array}{r}-293,5^{* * *} \\
(-7,69)\end{array}$ & $\begin{array}{r}-330, * * * \\
(-5,60)\end{array}$ & $\begin{array}{r}-321,5^{* * *} \\
(-5,42)\end{array}$ \\
\hline $\begin{array}{l}\text { West- bzw. Ostdeutsche } \\
\text { Kommune }\end{array}$ & $\begin{array}{r}-576,5^{* * *} \\
(-10,23)\end{array}$ & $\begin{array}{r}-658,5^{* * *} \\
(-6,45)\end{array}$ & $\begin{array}{r}-568,4 * * * \\
(-10,02)\end{array}$ & $\begin{array}{r}-727,4 * * * \\
(-7,47)\end{array}$ & $\begin{array}{r}-714,2 * * * \\
(-7,39)\end{array}$ \\
\hline $\begin{array}{l}\text { Kreisfreie Stadt }(1= \\
\text { kreisfrei) }\end{array}$ & $\begin{array}{r}535,83^{* * * *} \\
(7,66)\end{array}$ & $\begin{array}{r}502,35 * * * \\
(5,28)\end{array}$ & $\begin{array}{r}536,98^{* * * *} \\
(7,69)\end{array}$ & $\begin{array}{r}542,40^{* * * *} \\
(6,59)\end{array}$ & $\begin{array}{r}540,97 * * * \\
(6,59)\end{array}$ \\
\hline $\begin{array}{l}\text { Personalausgaben } \\
\text { Kernhaushalt }\end{array}$ & $\begin{array}{r}0,29 * * \\
(2,42)\end{array}$ & $\begin{array}{r}0,44^{* *} \\
(2,00)\end{array}$ & $\begin{array}{r}0,31^{* *} \\
(2,52)\end{array}$ & & \\
\hline $\begin{array}{l}\text { Gewerbesteuerauf- } \\
\text { kommen (netto) }\end{array}$ & $\begin{array}{c}-0,04 \\
(-0,59)\end{array}$ & $\begin{array}{c}-0,15 \\
(-1,57)\end{array}$ & & & \\
\hline $\begin{array}{l}\text { Kommunalverfassungs- } \\
\text { index }\end{array}$ & & $\begin{array}{r}-32,54 * * * \\
(-3,38)\end{array}$ & & $\begin{array}{r}-29,02^{* * * *} \\
(-3,38)\end{array}$ & $\begin{array}{r}-27,20^{* * *} \\
(-3,13)\end{array}$ \\
\hline $\begin{array}{l}\text { Größe des Gemeinde- } \\
\text { rates/Stadtparlaments }\end{array}$ & & $\begin{array}{c}0,36 \\
(0,12)\end{array}$ & & & \\
\hline $\begin{array}{l}\text { Zersplitterung Gemein- } \\
\text { derat (Rae-Index) }\end{array}$ & & $\begin{array}{l}-12,45 \\
(-0,04)\end{array}$ & & & \\
\hline $\begin{array}{l}\text { Index der Konsensde- } \\
\text { mokratie }\end{array}$ & & & $\begin{array}{l}-17,99 \\
(-1,006)\end{array}$ & & \\
\hline $\begin{array}{l}\text { SPD- } \\
\text { Oberbürgermeister }(=1 \text {, } \\
\text { sonst } 0)\end{array}$ & & & & $\begin{array}{l}50,90 \\
(1,04)\end{array}$ & \\
\hline $\begin{array}{l}\text { Sitzanteil CDU im } \\
\text { Gemeindeparlament }\end{array}$ & & & & $\begin{array}{c}-1,72 \\
(-0,79)\end{array}$ & $\begin{array}{l}-1,17 \\
(-0,52)\end{array}$ \\
\hline $\begin{array}{l}\text { Sitzanteil linker Partei- } \\
\text { en im Gemeindeparla- } \\
\text { ment }\end{array}$ & & & & & $\begin{array}{c}3,12 \\
(1,55)\end{array}$ \\
\hline Konstante & $\begin{array}{r}525,2^{* * * *} \\
(7,68)\end{array}$ & $\begin{array}{r}1050,6^{* * * *} \\
(4,41)\end{array}$ & $\begin{array}{r}515,3^{* * * *} \\
(7,49)\end{array}$ & $\begin{array}{r}1247,9^{* * * *} \\
(6,54)\end{array}$ & $\begin{array}{r}1098,4^{* * * *} \\
(4,87)\end{array}$ \\
\hline Fallzahl & 1170 & 730 & 1170 & 758 & 761 \\
\hline F-Statistik & $103,16^{* * *}$ & $55,83^{* * *}$ & $103,32 * * *$ & $66,532 * * *$ & $66,96 * * *$ \\
\hline Höchster VIF-Wert & 2,04 & 2,80 & 2,02 & 2,26 & 2,49 \\
\hline Korrigiertes $\mathrm{R}^{2}$ & 0,38 & 0,43 & 0,38 & 0,41 & 0,42 \\
\hline
\end{tabular}

Anmerkungen: Lineare Regression der Kassenkredite . Angegeben sind die Regressionskoeffizienten sowie in Klammern die t-Werte. Höchster VIF-Wert ist der höchste Varianzinflationsfaktor unter allen unabhängigen Variablen. ***: signifikant auf dem 1\%Niveau, **: signifikant auf dem 5\%-Niveau, *: signifikant auf dem 10\%-Niveau. Abhängige Variablen: Höhe der Kassenkredite in Euro pro Einwohner. Die unabhängigen Variablen sind auf das Jahr 2010 bezogen. 
In Gleichung 3 wird das Modell um den Index der Konsensdemokratie erweitert. Dieser Index wird additiv, entsprechend der Theorie von Lijphart, gebildet. Hierzu wird jeweils ein Punkt vergeben für einen starken Kommunalverfassungsindex (hier interpretiert als Balance zum Kommunalparlament), einen parteilosen Bürgermeister sowie eine hohe parteipolitische Zersplitterung im kommunalen Parlament. Dieser Index hat zwar das erwartete Vorzeichen (je konsensorientierter die Lokalpolitik desto niedriger die Kassenkredite), bleibt jedoch insignifikant. Die Konsensorientierung entsprechend des Lijphartschen Theoriegebäudes kann sicherlich durch weitere Variablen verbessert werden, was aktuell noch an den verfügbaren Daten auf kommunaler Ebene scheitert.

Die 4. Gleichung (vgl. Tab. 3) berücksichtigt neben den sozioökonomischen, den raumgeographischen und den institutionellen Variablen noch parteipolitische Faktoren. Dazu wird getestet, ob die Stadt durch einen SPD Bürgermeister regiert wird und wie die parteipolitische Färbung im Kommunalparlament aussieht. Es zeigt sich, dass weder ein SPD Bürgermeister (alternativ gilt gleiches für einen CDU Oberbürgermeister) einen signifikant dämpfenden oder erhöhenden Einfluss auf die Kassenkredite besitzt. Das positive Vorzeichen weist zwar darauf hin, dass SPD Oberbürgermeister einen eher erhöhenden Einfluss auf die Kommunalverschuldung haben, aber diese Variable ist statistisch nicht signifikant. Auch die Mehrheitsverhältnisse in den Kommunalparlamenten (vgl. Gleichung 4 und 5) sind nicht signifikant. Zwar weist ein hoher CDU-Anteil im Gemeindeparlament mit einem negativen Vorzeichen eher auf eine dämpfende Wirkung hin und der Anteil Linker Parteien eher auf einen erhöhenden Einfluss, jedoch sind diese Variablen wieder statistisch insignifikant.

Betrachtet man die Beta-Werte (nicht in Tabelle 3 ausgewiesen), die die relative Wichtigkeit der einzelnen Faktoren angeben, so sind die sechs wichtigsten Faktoren der Bestimmung der Kassenkredite:

(1) die Höhe der vorhandenen Gesamtverschuldung,

(2) der Anteil der Grundsicherungsempfänger (SGB II-Quote),

(3) die Region, d.h. handelt es sich um eine ost- oder westdeutsche Kommune,

(4) der Status als kreisfreie Stadt, der verschuldungserhöhend wirkt,

(5) die Bedeutung als Arbeitsort sowie

(6) der Kommunalverfassungsindex, der vor allem die Unabhängigkeit und Stellung der Bürgermeister misst.

Die endogenen Faktoren auf der kommunalen Ebene, also die Parteizugehörigkeit der Bürgermeister oder etwa auch der Sitzanteil einzelner Parteien, wie etwa 
der SPD, der CDU, oder der Wählergruppen sind stets insignifikant. Parteipolitische Faktoren spielen demnach, wenn man die anderen theoretisch relevanten Faktoren kontrolliert, keine Rolle. Exogene Faktoren sind die entscheidenden Einflussfaktoren zur Erklärung der Kassenkredite, zumindest im Querschnittsvergleich.

\section{Empirische Befunde von Regressionen mit Paneldaten}

Tabelle 4 zeigt die Ergebnisse einer Regressionsanalyse mit Paneldaten. Dabei wird die Veränderung der Kassenkredite durchgängig als abhängige Variable herangezogen.

Der Paneldatensatz umfasst die Jahre 2003 bis 2010. In der Betrachtung stehen Gemeinden mit einer Mindesteinwohnerzahl von 20.000. Diese Grenze wird gezogen, da die Verfügbarkeit der Daten darunter zunehmend abnimmt und die Heterogenität mit schrumpfender Bevölkerungszahl stark zunimmt. Die in Gleichung 1 aufgeführte Schätzgleichung soll Aufschluss über das verwendete Modell bringen.

\section{1. $\Delta$ Kassenkredite $_{\text {it }}=\beta_{1}$ Sozio $_{\text {it }}+\beta_{2}$ Pol $_{\text {it }}+$ $\beta_{3}$ KreisfreieStadtDummy $_{i t}+\alpha_{\mathrm{i}}+\gamma_{\mathrm{t}}+\mu_{\mathrm{it}}$}

Auf der rechten Seite der Gleichung stehen ausgewählte sozioökonomische Größen in Kommune i und im Jahr t. Diese sind die Bevölkerungszahl, welche gemessen in 1.000 Einwohnern in die Regressionen einfließt, da ansonsten deren Koeffizienten sehr klein würden. Die Erwerbstätigenquote findet als inverser Sozialbelastungsparameter Anwendung. Als Indikator für die Wirtschaftskraft soll die Variable Kaufkraft dienen. Außerdem wird der Zentralitätsparameter „Bedeutung als Arbeitsort“ in die Regressionsanalyse aufgenommen. Dieser ergibt sich aus Division aus den sozialversicherungspflichtig Beschäftigten am Arbeitsort und den sozialversicherungspflichtig Beschäftigten am Wohnort. Somit hat eine Kommune mehr Einpendler als Auspendler, wenn dieser Wert größer als 1 ist. Da die Werte dieser Größe im Minimum gegen 0 gehen und im Maximum bei 5 liegen sind hier besonders hohe Koeffizienten zu erwarten. Als Proxy für die Wirtschaftsstruktur einer Kommune fließt der Anteil der Erwerbstätigen im Dienstleistungssektor in die Regressionsanalyse ein. Zusätzlich könnte auch die Altersstruktur einer Kommune Auswirkungen auf deren Defizite haben. Somit wird auch der Altenquotient, welcher durch den Anteil der über 65Jährigen an den 20 bis 64-Jährigen definiert ist, berücksichtigt. 
Als weiterer Bestandteil der Schätzgleichung fließen diverse politökonomische Variablen $\left(\mathrm{Pol}_{\mathrm{it}}\right)$ in die Schätzungen ein. Hierbei handelt es sich um Parameter bezüglich der Zusammensetzung und den Eigenschaften des Gemeinde-/ Stadtrats: Die Größe des Gemeinderats, die Zersplitterung des Gemeindeparlaments (Rae-Index), die Wahltermine der Kommunalwahlen als Dummy-Variable, der CDU- sowie SPD-Sitzanteil im Stadtparlament sowie jeweils zwei DummyVariablen für die Parteizugehörigkeit des Bürgermeisters (CDU und SPD).

Da sich im Datensatz sowohl kreisangehörige Gemeinden als auch kreisfreie Städte befinden wird der kreisfreie Stadt Dummy als notwendige Kontrollvariable eingeführt. Damit wird der Tatsache Rechnung getragen, dass heterogene Aufgabenbelastungen zugrunde liegen. So sind beispielsweise die kreisfreien Städte und Landkreise Hauptträger der Finanzierung sozialer Leistungen. ${ }^{14}$ Dementsprechend findet dieser Dummy in allen Schätzungen Anwendung.

Starke Heterogenität zwischen den Flächenländern besteht beispielsweise durch den Kommunalisierungsgrad eines Landes, also dem Anteil öffentlicher Aufgaben bzw. Ausgaben, der durch die kommunale Ebene ausgeführt wird ${ }^{15}$ oder auch durch den haushaltsrechtlichen Ordnungsrahmen eines Landes. ${ }^{16}$ Um generell solche flächenlandspezifische Unterschiede, die zum Teil nicht beobachtbar oder messbar sind, aber Einfluss auf die abhängige Variable haben können, zu kontrollieren, werden in allen Spezifikationen fixe Flächenlandeffekte verwendet. Ansonsten droht eine Verfälschung der Ergebnisse, da die Schätzergebnisse aufgrund Vernachlässigung solcher Unterschiede und weiterer Faktoren, die mit der abhängigen und einer oder mehreren unabhängigen Variablen zusammenhängen, unter- oder überschätzt werden können (Omitted Variable Bias). ${ }^{17}$ In der Gleichung 1 steht $\alpha_{\mathrm{i}}$ für fixe Effekte für Flächenländer. $\alpha_{\mathrm{i}}$ kontrolliert also zeitlich invariante Unterschiede zwischen den Flächenländern, welche nicht durch die verwendeten Variablen beobachtet werden können. ${ }^{18}$

Zusätzlich dazu werden fixe Zeiteffekte verwendet. Hierdurch sollen kommuneneinheitliche Unterschiede über die Zeit kontrolliert werden. Hiermit werden

14 Vgl. Junkernheinrich, M.: Kommunale Sozialleistungen im Ländervergleich. Ein Betrag zur finanzpolitischen Berichterstattung, in: Zeitschrift für Staats- und Europawissenschaften, 2 (2012).

15 Vgl. Junkernheinrich, M.: Die fiskalische Einbindung der Kommunen in den deutschen Bundesländern, in: Junkernheinrich, M. et al. (Hg.): Jahrbuch für öffentliche Finanzen 2009, Berlin, 2009, 195 - 220.

16 Vgl. Frankenberg, D./Junkernheinrich, M.: Kommunalfinanzen im Jahre 2013: Der lange Weg zur Konsolidierung, in: Junkernheinrich, M. et al. (Hg.): Jahrbuch für öffentliche Finanzen 2014, Berlin, 2014, 299-321.

17 Vgl. Stock, J. H./ Watson, M. W.: Introduction to Econometrics, Boston, 2003.

18 Vgl. Greene, W. H.: Econometric Analysis, New York, 2008, 193-199. 
beispielsweise bundesweite Schocks oder jahresspezifische Veränderungen aufgefangen. $\gamma_{\mathrm{t}}$ steht in der Gleichung für die verwendeten fixen Zeiteffekte. ${ }^{19}$

Da Heteroskedastizität und auch Autokorrelation der Fehlerterme $(\mu)$ für das vorliegende Panel nicht ausgeschlossen werden können, werden robuste und geclusterte Standardfehler auf Ebene der Kommunen in allen Spezifikationen verwendet ${ }^{20}$. Somit wird für eine potentielle Korrelation der Fehlerterme innerhalb einer Kommune über die Zeit kontrolliert.

Tabelle 4 zeigt in allen Spalten Schätzungen mit der Veränderung der Kassenkreditverschuldung als abhängige Variable. In den Spalten 1 bis 3 werden unterschiedliche politische Variablen nacheinander eingeführt. So soll beispielsweise die Zersplitterung der Gemeinderäte in keine gemeinsame Schätzung mit den Sitzanteilen CDU/SPD, da diese schließlich Bestandteile des Index sind. In Spalte 4 wird zusätzlich der Einfluss des Kommunalverfassungsindex überprüft. Dafür werden in Schätzung 4 die fixen Flächenlandeffekte ausgelassen, da der Index flächenlandspezifisch ist und nicht über die Zeit variiert. Im Folgenden werden zunächst die Ergebnisse der Schätzungen 1 bis 3 diskutiert, da diese aufgrund der fixen Effekte präziser sein sollten. Für den Einfluss des Kommunalverfassungsindex wird im Anschluss Schätzung 4 interpretiert.

Die Variablen Erwerbstätigenquote und Erwerbstätigenanteil im Dienstleistungssektor sind in den betrachteten Spezifikationen insignifikant bzw. nur sehr schwach signifikant (H1c). Die Koeffizienten beider Variablen haben durchgehend ein negatives Vorzeichen, wirken also von der Tendenz her verschuldungshemmend. Ansonsten zeigt sich ein deutlicher Einfluss der verwendeten sozioökonomischen Variablen. So wirken sich die Kaufkraft und die Bedeutung als Arbeitsort stark negativ signifikant auf die Veränderung der Kassenkredite aus (H1c und H1d). Eine Erhöhung der Kaufkraft um 1.000 Euro führt hiernach zu einem um 2 Euro je Einwohner schwächeren Anstieg der Kassenkredite, während eine um 0,1 höhere Bedeutung als Arbeitsort mit um ca. 3,5 bis 3,8 Euro je Einwohner schwächer steigenden Kassenkrediten einhergeht (vgl. Spalten 1 bis 3). Demgegenüber wirkt der Altenquotient verschuldungstreibend (H1b). Dessen Koeffizient ist durchgehend signifikant auf dem $1 \%$-Niveau. Je älter also eine Kommune, desto stärker steigen deren Kassenkredite. Die Bevölkerungszahl hat

19 Vgl. Greene, W., a.a.O.

20 Vgl. Petersen, M. A.: Estimating Standard Errors in Finance Panel Data Sets: Comparing Approaches, in: The Review of Financial Studies, Oxford, 2009, 475-476. 
einen schwach negativ signifikanten Einfluss auf die Veränderung der Kassenkredite $(\mathrm{H} 1 \mathrm{a})$.

Bei den politökonomischen Größen zeigen sich die Sitzanteile CDU/SPD als insignifikant. Zu erwähnen ist jedoch, dass sich das nach Hypothese 4a erwartet positive Vorzeichen für SPD-Sitzanteile (von der Tendenz her schuldentreibend) und nach Hypothese $4 \mathrm{~b}$ das erwartet negative Vorzeichen für CDU-Sitzanteile (von der Tendenz her schuldensenkend) einstellt. Jedoch sind nur Aussagen über die Tendenz möglich und auch die Hypothesen können nicht nachhaltig bestätigt werden, da alle Koeffizienten insignifikant sind.

Demgegenüber können die Hypothesen 5a (Gehört der Bürgermeister einer Partei des linken Spektrums (SPD, Linke oder Grüne) an, desto stärker verschuldet sich die Kommune)) und 5b (Gehört der Bürgermeister einer bürgerlichen Partei (CDU/CSU, FDP) an, desto weniger verschuldet sich die Kommune) bestätigt werden. In Schätzung 1 hat die Dummy-Variable für den CDU-Bürgermeister einen signifikanten Einfluss auf dem 5 \%-Niveau. Das Vorzeichen des Koeffizienten ist negativ. Somit steigt in Kommunen, deren Bürgermeister der CDU angehört, die Verschuldung durch Kassenkredite im Durchschnitt um ca. 11 Euro je Einwohner schwächer als in Kommunen mit Bürgermeister anderer Parteizugehörigkeit. Noch stärker signifikant ist der Einfluss der Dummy-Variable für SPD-Bürgermeister. Auch der Koeffizient dieser Variablen ist etwas höher. So steigt in Kommunen mit SPD-Bürgermeister die Kassenkreditverschuldung stärker an als in Kommunen mit Bürgermeistern anderer Parteizugehörigkeit.

Die Größe des Gemeindeparlaments übt durchgehend einen positiven Einfluss auf die Veränderung der Kassenkredite aus. Der Einfluss ist signifikant auf dem $5 \%$-Niveau. Nach Schätzungen 1 bis 3 steigen die Kassenkredite um ca. 1 Euro je Einwohner stärker an, wenn sich das Stadtparlament um ein Ratsmitglied erhöht. Da ein Zusammenhang zwischen der Bevölkerungszahl einer Kommune und deren Gemeinderatsgröße besteht, wird bewusst die Bevölkerungszahl in den selben Schätzungen eingeschlossen, um auszuschließen, dass die Variable Gemeindegröße lediglich einen Größeneffekt beschreibt. Die Korrelation dieser beiden Größen liegt bei $65 \%$, jedoch ist auch nach einschlägigen Tests $^{21}$ keine erhöhte Kollinearität zu erwarten. Zudem bleiben ca. 35 \% beobachtete Abweichungen der beiden Variablen, die das Modell präzisieren können.

21 Getestet wurden die VIF-Werte der Schätzungen, diese sind mit maximal ca. 4 immer deutlich unterhalb des kritischen Wertes von 10. Vgl. hierzu Wooldridge, J. M.: Introductory Econometrics, Mason, 2013. 
In Spalte 3 wird die Zersplitterung des Gemeindeparlaments als Rae-Index eingeführt. Es zeigt Signifikanz auf dem 5 \%-Niveau beim entsprechenden Koeffizienten. Hiernach gilt: Erhöht sich der Index um 0,1 so steigen die Kassenkredite um ca. 9,7 Euro je Einwohner stärker. Je zersplitterter also der Gemeinderat, desto stärker steigt die Verschuldung durch Kassenkredite.

Hypothese 6 thematisiert den Zusammenhang zwischen Verschuldung und dem Zeitraum vor Kommunalwahlen. Die Hypothese, dass in der Zeit vor Kommunalwahlen die Verschuldung stärker ansteigt, kann in den vorliegenden Schätzungen nicht bestätigt werden. Der Koeffizient für die Dummy-Variable für Kommunalwahljahre ist schwach signifikant (auf dem $10 \%$-Niveau). Dieser hat jedoch ein negatives Vorzeichen, was eher für weniger Zusatzverschuldung in Wahljahren spricht. Dieses Ergebnis ist allerdings aufgrund des für diesen Zweck sehr geringen Beobachtungszeitraums auch nur beschränkt aussagekräftig.

In Schätzung 4 fällt der hochsignifikante Einfluss der Kommunalverfassung auf. Je stärker die institutionelle Stellung von Bürgermeistern ist, desto geringer ist der Anstieg der Verschuldung durch Kassenkredite. Dieses Ergebnis entspricht der hierzu aufgestellten Hypothese 7. Die politischen Variablen weisen in den Schätzungen teilweise recht hohe Signifikanzniveaus auf. In den Spezifikationen wurde versucht anhand der ausgewählten Variablen sowie der Verwendung von fixen Flächenland und Zeiteffekten zu verhindern, dass Variablen fehlen, die Determinanten der abhängigen Variablen und zugleich korreliert mit den entsprechenden politischen Variablen sind (oben beschriebenes Problem des Omitted Variable Bias). 
Tabelle 4: Panelregressionsmodell zur Erklärung der Verschuldung durch Kassenkredite

\begin{tabular}{|c|c|c|c|c|}
\hline Variablen & $\begin{array}{c}(1) \\
\Delta \text { Kassenkre- } \\
\text { dite }\end{array}$ & $\begin{array}{c}(2) \\
\Delta \text { Kassenkre- } \\
\text { dite }\end{array}$ & $\begin{array}{c}(3) \\
\Delta \text { Kassenkre- } \\
\text { dite }\end{array}$ & $\begin{array}{c}(4) \\
\Delta \text { Kassenkre- } \\
\text { dite } \\
\end{array}$ \\
\hline Bevölkerung & $\begin{array}{l}-0,072^{*} \\
(-1,814)\end{array}$ & $\begin{array}{l}-0,072^{*} \\
(-1,840)\end{array}$ & $\begin{array}{c}-0,066 \\
(-1,628)\end{array}$ & $\begin{array}{c}-0,162^{* * * *} \\
(-3,353)\end{array}$ \\
\hline Erwerbstätigenquote & $\begin{array}{l}-0,940 \\
(-1,451)\end{array}$ & $\begin{array}{l}-1,090^{*} \\
(-1,677)\end{array}$ & $\begin{array}{l}-0,815 \\
(-1,248)\end{array}$ & $\begin{array}{l}-1,287 * * \\
(-1,981)\end{array}$ \\
\hline Kaufkraft & $\begin{array}{c}-0,002 * * * \\
(-3,126)\end{array}$ & $\begin{array}{c}-0,002^{* * * *} \\
(-3,038)\end{array}$ & $\begin{array}{c}-0,002^{* * * *} \\
(-3,845)\end{array}$ & $\begin{array}{c}-0,001 * * \\
(-2,330)\end{array}$ \\
\hline Bedeutung als Arbeitsort & $\begin{array}{c}-35,180^{* * *} \\
(-4,570)\end{array}$ & $\begin{array}{c}-35,111^{* * *} \\
(-4,537)\end{array}$ & $\begin{array}{c}-37,776^{* * *} \\
(-4,941)\end{array}$ & $\begin{array}{c}-37,042 * * * \\
(-4,708)\end{array}$ \\
\hline $\begin{array}{l}\text { Erwerbstätigenanteil im } \\
\text { Dienstleistungssektor }\end{array}$ & $\begin{array}{l}-0,260 \\
(-1,089)\end{array}$ & $\begin{array}{l}-0,210 \\
(-0,901)\end{array}$ & $\begin{array}{c}-0,272 \\
(-1,122)\end{array}$ & $\begin{array}{l}-0,531 * * \\
(-2,512)\end{array}$ \\
\hline Altenquotient & $\begin{array}{c}2,144 * * * \\
(3,090)\end{array}$ & $\begin{array}{c}2,280^{* * *} \\
(3,265)\end{array}$ & $\begin{array}{c}2,280 * * * \\
(3,343)\end{array}$ & $\begin{array}{c}1,705^{* * *} \\
(2,900)\end{array}$ \\
\hline kreisfreie Stadt $(0 / 1)$ & $\begin{array}{c}62,871^{* * *} \\
(4,293)\end{array}$ & $\begin{array}{c}62,306^{* * *} \\
(4,267)\end{array}$ & $\begin{array}{c}62,244 * * * \\
(4,217)\end{array}$ & $\begin{array}{c}64,394 * * * \\
(3,812)\end{array}$ \\
\hline $\begin{array}{l}\text { Größe des Gemeindera- } \\
\text { tes/Stadtparlaments }\end{array}$ & $\begin{array}{l}1,153^{* *} \\
(2,537)\end{array}$ & $\begin{array}{l}1,145^{* *} \\
(2,533)\end{array}$ & $\begin{array}{l}1,071 * * \\
(2,364)\end{array}$ & $\begin{array}{c}2,615 * * * \\
(5,695)\end{array}$ \\
\hline $\begin{array}{l}\text { CDU-Bürgermeister } \\
(0 / 1)\end{array}$ & $\begin{array}{c}-11,051^{* *} \\
(-2,044)\end{array}$ & & & \\
\hline $\begin{array}{l}\text { CDU-Sitzanteil im } \\
\text { Gemeinde- } \\
\text { rat/Stadtparlament }\end{array}$ & $\begin{array}{c}-0,449 \\
(-1,633)\end{array}$ & & & \\
\hline SPD-Bürgermeister $(0 / 1)$ & & $\begin{array}{c}16,051^{* * * *} \\
(2,824)\end{array}$ & & \\
\hline $\begin{array}{l}\text { SPD-Sitzanteil im Ge- } \\
\text { meinderat/Stadtparlament }\end{array}$ & & $\begin{array}{c}0,049 \\
(0,142)\end{array}$ & & \\
\hline $\begin{array}{l}\text { Zersplitterung des Ge- } \\
\text { meindeparlaments (Rae- } \\
\text { Index) }\end{array}$ & & & $\begin{array}{c}96,672^{* *} \\
(2,492)\end{array}$ & \\
\hline Kommunalwahljahr (0/1) & & & $\begin{array}{l}-8,808^{*} \\
(-1,726)\end{array}$ & \\
\hline $\begin{array}{l}\text { Kommunalverfassungs- } \\
\text { index }\end{array}$ & & & & $\begin{array}{c}-3,689 * * * \\
(-3,578)\end{array}$ \\
\hline Konstante & $\begin{array}{l}91,070 \\
(1,583) \\
\end{array}$ & $\begin{array}{l}66,643 \\
(1,253) \\
\end{array}$ & $\begin{array}{l}15,038 \\
(0,284) \\
\end{array}$ & $\begin{array}{c}147,399 * * * \\
(3,080)\end{array}$ \\
\hline Beobachtungen & & 4.444 & 4.448 & 4.450 \\
\hline Korrigiertes $\mathrm{R}^{2}$ & 0,163 & 0,163 & 0,162 & 0,123 \\
\hline
\end{tabular}

Anmerkungen: Spalten 1-3: OLS-Schätzungen mit fixen Zeit- und Flächenlandeffekten; Spalte 4: OLS-Schätzung mit fixen Zeiteffekten; in den Klammern sind t-Statistiken gegeben; Standardfehler sind robust und 'geclustert' nach Kommunen; die Sternchen kennzeichnen Signifikanz auf dem $10\left(^{*}\right), 5(* *)$ und $1(* * *)$ Prozent-Niveau. 
So ist beispielsweise denkbar, dass besonders in Kommunen mit einer hohen sozialen Belastung vermehrt SPD-Bürgermeister gewählt wurden und nicht die Parteizugehörigkeit des Bürgermeisters sondern die Sozialbelastung den Ausschlag für die zusätzliche Verschuldung gegeben hat. Aus diesem Grund wurden die Schätzungen auch unter Einbeziehung der SGB II-Quote, die über weniger Jahre vorliegt und deshalb in den dargestellten Schätzungen nicht berücksichtigt wird, durchgeführt. Dies änderte jedoch nichts an den Ergebnissen bezüglich der Parteizugehörigkeit. ${ }^{22}$ Dennoch ist das Problem fehlender erklärender Variablen natürlich nie vollständig auszuschließen.

\section{Politische Optionen zur Lösung des Kassenkreditproblems}

Die Kommunen, Land und Bund können je nach Kompetenzrahmen, politischen Präferenzen und zur Verfügung stehenden finanziellen Mitteln unterschiedlich auf hohe Kassenverstärkungskredite reagieren. Von privater Seite besteht die Möglichkeit, dass auch die Kreditwirtschaft als finanzierender Akteur ihr Verhalten ändert, was sie in den letzten Jahren auch verstärkt gemacht hat (vgl. Abb. 3). Im Folgenden liegt der Fokus jedoch vor allem auf der staatlichen Ebene, da die Problemlösung schwerpunktmäßig hier zu leisten ist.

22 Auf Anfrage können die Ergebnisse mit inkludierter SGB II-Quote selbstverständlich nachgereicht werden. 
Abbildung 3: Mögliche Ansätze zum Abbau von Kassenkrediten nach Akteuren im Überblick

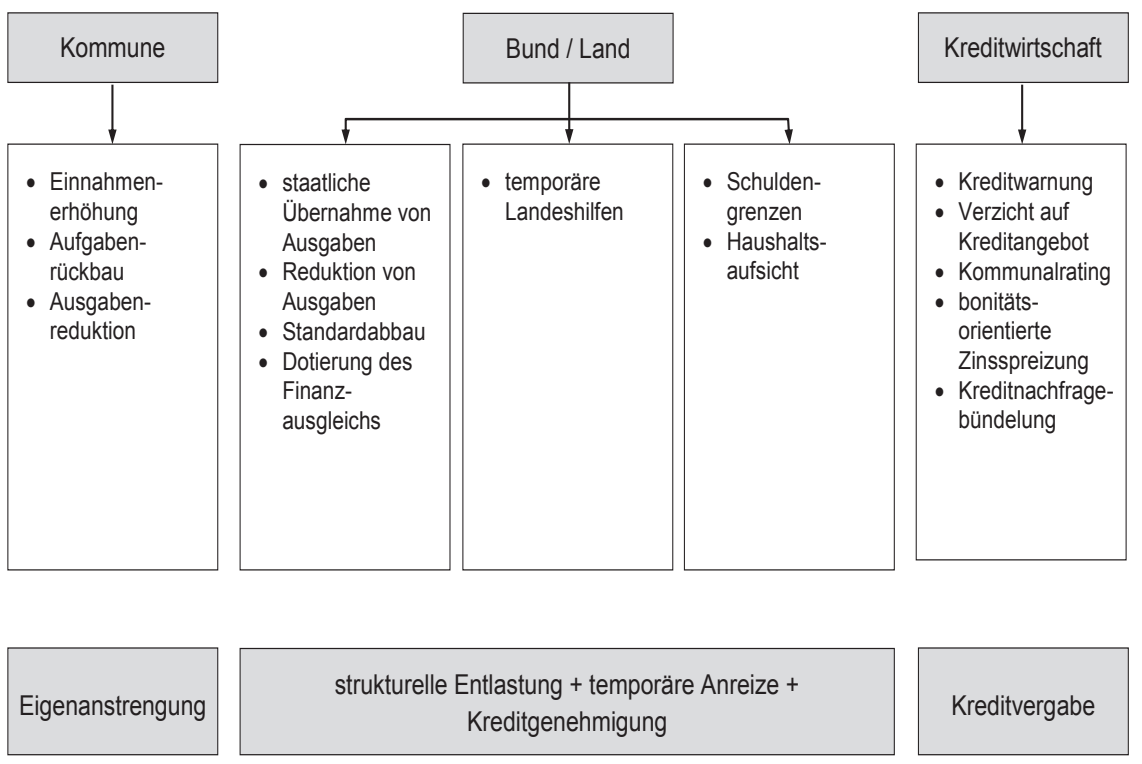

Anmerkung: Eigene Darstellung

\section{Maßnahmen der Kommunen}

Die instrumentalen Möglichkeiten der Kommunen, also eigenständige Maßnahmen, sind für das Ziel ausgeglichener Haushalte bzw. nachhaltiger Finanzen in den folgenden Bereichen zu finden:

- Erhöhung der eigenen Einnahmen,

- Reduzierung der Ausgaben,

- Rückbau von Aufgaben,

- Benchmarking, Kennzahlenvergleiche und Frühwarnsystem sowie

- $\quad$ organisatorische und institutionelle Maßnahmen.

Eine Möglichkeit der Informationsgewinnung ist es, die verantwortlichen Bürgermeister zu befragen, welche Möglichkeiten sie selbst als verantwortlich handelnde Personen präferieren. Hier kann u.a. nach dem Handlungsspielraum von Steuererhöhungen auf Gemeindeebene gefragt werden. Betrachtet man die Gesamtheit aller Kommunen, so wurden in den letzten Jahren die Gewerbesteuer 
(als wichtigste kommunale Steuer) und die Grundsteuer stetig erhöht. Es spricht einiges dafür, dass sich dieser Trend fortsetzen wird. ${ }^{23}$

Die Bürgermeister wurden etwa befragt, ob die ökonomische Situation in ihrer Kommune eine Anhebung des Hebesatzes in der Grundsteuer B sowie eine Anhebung des Hebesatzes der Gewerbesteuer erlauben würde (vgl. Tab. 5 und 6). Die überwiegende Mehrheit der befragten Bürgermeister sieht bei der Grundsteuer B einen Handlungsspielraum (67,9\%). Bemerkenswert ist allerdings die unterschiedliche Verteilung zwischen den west- und ostdeutschen Kommunen. In den ostdeutschen Kommunen sieht die Hälfte der Bürgermeister keinen Handlungsspielraum, während in Westdeutschland immerhin knapp $71 \%$ der Bürgermeister noch einen Handlungsspielraum bei der Grundsteuer B sieht. Kontrastiert man dies mit den tatsächlichen Hebesätzen in Ost- und Westdeutschland, überrascht dies, denn die Hebesätze bei der Grundsteuer B (also für bebaute Grundstücke) liegen in Westdeutschland höher als in Ostdeutschland.

Tabelle 5: Einschätzung des Handlungsspielraumes einer Erhöhung des Hebesatzes bei der Grundsteuer B

\begin{tabular}{c|llccc}
\hline \multicolumn{2}{c}{} & & $\begin{array}{c}\text { Ost- } \\
\text { deutschland }\end{array}$ & $\begin{array}{c}\text { West- } \\
\text { deutschland }\end{array}$ & Summe \\
\hline & Anzahl & 55 & 204 & 259 \\
\hline Summe & & An $\%$ & $50,5 \%$ & $29,2 \%$ & $32,1 \%$ \\
\hline & Anzahl & 54 & 494 & 548 \\
\hline
\end{tabular}

Quelle: Bürgermeisterbefragung 2014 (Wagschal/Grasl 2014), Fragetext: Würde die aktuelle ökonomische Situation in Ihrer Kommune eine Anhebung des Hebesatzes der Grundsteuer B erlauben? Dargestellt sind Spaltenprozente.

23 Vgl. Wagschal, U./ Grasl, M.: Kommunale Haushaltspolitik. Ergebnisse einer Befragung der Bürgermeister Deutschlands, Freiburg, 2014. Insgesamt konnte bei der Bürgermeisterbefragung ein Rücklauf von Antworten aus 863 Kommunen erzielt werden. Dies ist ein für sozialwissenschaftliche Umfragen vergleichsweise hoher Anteil und damit ein sehr zufriedenstellendes Ergebnis. Bezogen auf die Grundgesamtheit von 1.572 deutschen Kommunen, die mehr als 10.000 Einwohner (Stand 31.1.2010) aufweisen, ergibt sich eine Rücklaufquote von $54,9 \%$. 
Tabelle 6: Einschätzung des Handlungsspielraumes einer Erhöhung des Hebesatzes bei der Gewerbesteuer

\begin{tabular}{l|llrrr}
\hline \multicolumn{2}{c}{} & \multicolumn{2}{c}{$\begin{array}{l}\text { Ostdeutsch- } \\
\text { land }\end{array}$} & $\begin{array}{l}\text { West- } \\
\text { deutschland }\end{array}$ & Summe \\
\hline & & Anzahl & 70 & 306 & 376 \\
\hline & Nein & In \% & $64,8 \%$ & $44,0 \%$ & $46,8 \%$ \\
\hline & Anzahl & 38 & 390 & 428 \\
\hline & & In \% & $35,2 \%$ & $56,0 \%$ & $53,2 \%$ \\
\hline
\end{tabular}

Quelle: Bürgermeisterbefragung 2014, ${ }^{24}$ Fragetext: Würde die aktuelle ökonomische Situation in Ihrer Kommune eine Anhebung des Hebesatzes der Gewerbesteuer erlauben? Dargestellt sind Spaltenprozente.

Die Situation für die Gewerbesteuer weicht etwas ab (vgl. Tab. 6). Hier ist der Anteil derjenigen, die einen Spielraum für eine Anhebung der Gewerbesteuer sehen, in Gesamtdeutschland bei nur $53 \%$ aller Bürgermeister, also rund 15 Prozentpunkte geringer als bei der Grundsteuer. Wiederum gibt es einen OstWest-Unterschied, der jedoch nicht so deutlich ausgeprägt ist wie bei der Grundsteuer. In Westdeutschland sehen $56 \%$ der Bürgermeister einen Spielraum zur Erhöhung der Gewerbesteuer, in Ostdeutschland nur rund $35 \%$ der Bürgermeister, dementsprechend sieht eine Mehrheit keinen weiteren Handlungsspielraum bei der Gewerbesteuer in Ostdeutschland (65\% gegenüber $44 \%$ bei westdeutschen Kommunen). Zudem zeigen weitere Auswertungen, dass kleinere Städte eher einen Erhöhungsspielraum sehen als größere Städte, die jedoch wiederum höhere soziale Lasten tragen.

Die Bürgermeisterumfrage 2014 hat zudem ergeben, ${ }^{25}$ dass von der Mehrzahl der Bürgermeister eine zu geringe Finanzausstattung der Kommunen als Problem bezeichnet wird. Haushaltspolitik ist - vor allem auch auf kommunaler Ebene zu einem Gutteil exogen beeinflusst. Dennoch gibt es auch auf der Handlungs- 
ebene zahlreiche Maßnahmen, die eine Entlastungswirkung haben können. Die Gemeindeprüfungsanstalt Nordrhein-Westfalen (GPA) hat 2013 die verschiedenen Sparmaßnahmen der Kommunen empirisch erfasst und über 4.000 solcher Maßnahmen erhoben. Diese rund 4.000 Maßnahmen wurden dann zu rund 800 Maßnahmen zusammengefasst (vor allen Dingen wurden Redundanzen bereinigt), so dass diese Liste als Blaupause für die Haushaltskonsolidierung gelten kann. Fasst man die Maßnahmen nach ihrem Anteil am Konsolidierungsvolumen zusammen, so ergeben sich interessante Parallelen zu den empirischen Befunden in den Tabellen 5 und 6. Am häufigsten wurde die Grundsteuer B erhöht, gefolgt von einer Erhöhung des Gewerbesteueraufkommens. Als dritthäufigste Maßnahme wurde Personalabbau durchgeführt und als viertes Organisationsveränderungen in der Verwaltung. Weitere Maßnahmen waren Änderungen der Kinder-, Jugend- und Familienhilfe sowie die Erhöhung von Gewinnabführungen aus Beteiligungen. ${ }^{26}$ Bertelsmann Stiftung (Hg.): Städte in Not? Wege aus der Schuldenfalle, Gütersloh, 2013, hier 1563. 
Abbildung 4: Erfolgreiche Konsolidierungsmaßnahmen aus der Sicht der Bürgermeister

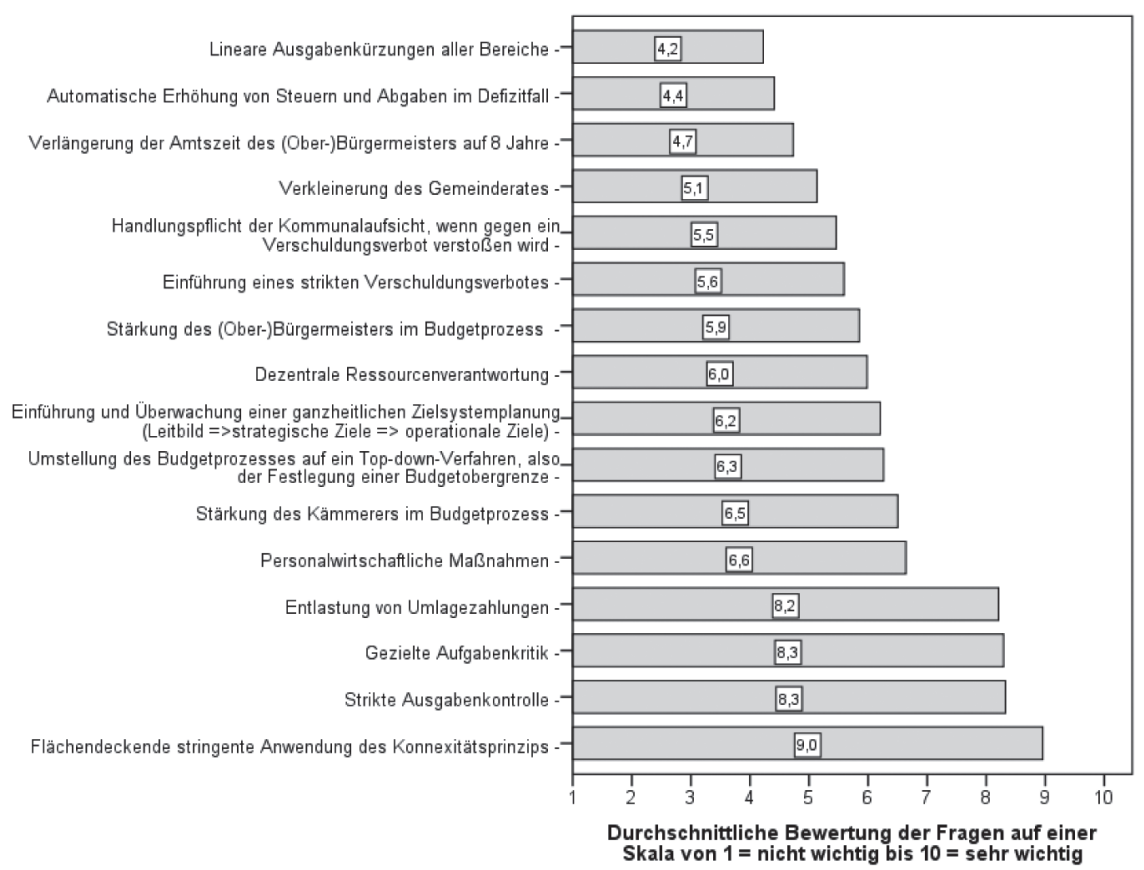

Quelle: Bürgermeisterbefragung 2014. Fragetext: „Wie beurteilen Sie den Beitrag folgender ausgewählter Möglichkeiten zu einer erfolgreichen Haushaltskonsolidierung? Weisen Sie den Themen Punkte entsprechend deren Nützlichkeit zu $(1=$ nicht hilfreich, ..., $10=$ sehr hilfreich); $\mathrm{n} \max =710$ für diese Frage.

Schließlich wurden in der Bürgermeisterbefragung 2014 die Bürgermeister gefragt, welche Maßnahmen sie als besonders erfolgreich zur Haushaltskonsolidierung ansehen. Neben der Stärkung des Konnexitätsprinzips wurden eine strikte Ausgabenkontrolle sowie eine gezielte Aufgabenkritik und die Entlastung von Umlagezahlungen genannt (vgl. Abb. 4). Im Mittelfeld landen Maßnahmen, die den Budgetprozess stringenter ausgestalten, wie etwa die Umstellung auf ein Top-Down-Verfahren sowie die Stärkung des Kämmerers und des Bürgermeisters. Besonders harte Maßnahmen wie eine lineare Ausgabenkürzung oder die automatische Erhöhung von Steuern und Abgaben, die man etwa in einzelnen Kantonen in der Schweiz kennt, werden überwiegend und deutlich abgelehnt.

Im Wesentlichen sind es also einnahmen- und ausgabenorientierte Maßnahmen, die zur Haushaltskonsolidierung geführt wurden. Innovativere Verfahren wie 
Benchmarking und Kennzahlenvergleiche ${ }^{27}$ werden dagegen weniger verwendet. Ein wichtiger Schritt, analog zum Vorgehen des Stabilitätsrates auf Länderebene, wäre die Implementation eines indikatorgestützten Frühwarnsystems. ${ }^{28}$

\section{Maßnahmen der Länder}

Verfassungspolitisch sind die Kommunen Teil der Länder. Daher haben die Länder eine besondere Verantwortung bei der Sicherstellung einer aufgabenangemessenen Finanzausstattung und der Lösung des Kassenkreditproblems. Eine Möglichkeit seitens der Bundesländer den besonders verschuldeten Kommunen zu helfen, sind kommunale Entschuldungsprogramme oder sogenannte Stärkungspakte. ${ }^{29}$ Mittlerweile haben zehn von dreizehn Flächenländern solche Programme (vgl. Tab. 7). Lediglich Baden-Württemberg, Sachsen und Thüringen haben keine solchen Programme.

Die Reichweite, die Dauer und auch der monetäre Umfang variieren zwischen den Bundesländern deutlich. Die aktuell umfangreichsten Programme finden sich in Nordrhein-Westfalen (knapp 6 Mrd.) sowie Rheinland-Pfalz und Hessen. Hier zeigt sich schon, dass diejenigen Bundesländer, die besonders hohe kommunale Kassenkredite haben, auch eher solche Programme auflegen. Die Ziele dieser Programme (vgl. Tab. 8) sind vor allen Dingen die Haushaltskonsolidierung, die Schuldentilgung aber auch die Gewährung von Zins- und Tilgungshilfen, um die Verschuldungsproblematik besser in den Griff zu bekommen. Die Finanzierung dieser Maßnahmen findet in mehr als der Hälfte der Länder ausschließlich durch das Land statt. Allerdings werden in Nordrhein-Westfalen, Rheinland-Pfalz und Niedersachsen sowie Schleswig-Holstein auch die Kommunen einerseits über den kommunalen Finanzausgleich (KFA), aber u.a. auch im Falle von Rheinland-Pfalz direkt über einen Eigenanteil der teilnehmenden Kommunen an dieser Entschuldung mit beteiligt.

27 Vgl. Bogumil, J. / Jann, W.: Verwaltung und Verwaltungswissenschaft in Deutschland. Einführung in die Verwaltungswissenschaft, 2. Auflage, Wiesbaden. 2009.; Thau, A.: Benchmarking in öffentlichen Verwaltungen, Berlin, 2009.

28 Vgl. Hesse, M. / Woitik, F.: Frühwarnsysteme - ein innovativer Beitrag zur Sicherung der kommunalen Haushaltsstabilität, in: Bertelsmann Stiftung (Hg.): Städte in Not?, Wege aus der Schuldenfalle, Gütersloh, 2013, 164-190.

29 Vgl. Stolzenberg, P. / Huber, H.: ,Die Griechen von NRW‘. Kommunale Rettungsschirme der Bundesländer, in: Der Moderne Staat, 6/2 (2013), 463-484. 
Tabelle 7: Kommunale Stärkungspakte und Entschuldungsprogramme der Länder

\begin{tabular}{|c|c|c|c|c|c|}
\hline Land & Name & $\begin{array}{l}\text { Inkraft- } \\
\text { treten }\end{array}$ & $\underset{2}{\text { Anzahl }}$ & Laufzeit & $\begin{array}{l}\text { (Gesamt-) Um- } \\
\text { fang in Mrd. } \\
\text { Euro, wenn nicht } \\
\text { anders gekenn- } \\
\text { zeichnet }\end{array}$ \\
\hline Bayern & $\begin{array}{l}\text { „Struktur- bzw. } \\
\text { Konsolidierungs- } \\
\text { hilfen“ (befristet) }\end{array}$ & 2007 & 32 & $\begin{array}{c}\text { Höchstens } 5 \\
\text { Jahre pro } \\
\text { Kommune }\end{array}$ & $\begin{array}{l}\text { Jahresvolumen: } \\
\text { 0,01 Mrd. Euro }\end{array}$ \\
\hline $\begin{array}{l}\text { Branden- } \\
\text { burg }\end{array}$ & Ausgleichsfonds & 2001 & 222 & - & $\begin{array}{l}\text { Von } 2013-2015 \text { je } \\
0,045 \mathrm{Mrd} \text {. Euro } \\
\text { pro Jahr, ab } 2016 \\
0,04 \mathrm{Mrd} \text {., bis } \\
2013 \text { insgesamt } \\
0,387 \text { Mrd. Euro } \\
\text { zugewiesen }\end{array}$ \\
\hline Hessen & $\begin{array}{l}\text { Kommunaler } \\
\text { Rettungsschirm }\end{array}$ & 2012 & 106 & 2013-2017 & 3,2 \\
\hline $\begin{array}{l}\text { Mecklen- } \\
\text { burg- } \\
\text { Vorpom- } \\
\text { mern }\end{array}$ & $\begin{array}{l}\text { Kommunaler } \\
\text { Konsolidierungs- } \\
\text { fonds }\end{array}$ & 2012 & & $2012-2015$ & 0,137 \\
\hline $\begin{array}{l}\text { Nieder- } \\
\text { sachsen }\end{array}$ & $\begin{array}{l}\text { Entschuldungs- } \\
\text { fonds (Teil des } \\
\text { „Zukunftsvertra- } \\
\text { ges“) }\end{array}$ & 2012 & & 2012-2029 & 1,26 \\
\hline $\begin{array}{l}\text { Nord- } \\
\text { rhein- } \\
\text { Westfalen }\end{array}$ & $\begin{array}{l}\text { Stärkungspakt } \\
\text { Stadtfinanzen }\end{array}$ & 2012 & 61 & 2012-2020 & 5,85 \\
\hline $\begin{array}{l}\text { Rhein- } \\
\text { land-Pfalz }\end{array}$ & $\begin{array}{l}\text { Kommunaler } \\
\text { Entschuldungs- } \\
\text { fonds (KEF-RP) }\end{array}$ & 2012 & & 2012-2026 & 3,825 \\
\hline Saarland & $\begin{array}{l}\text { Entschuldungs- } \\
\text { fonds Kommunen } \\
21\end{array}$ & 2013 & & 2013-2020 & $\begin{array}{l}\text { Jährlich rund } \\
0,017 \text { Mrd. Euro }\end{array}$ \\
\hline $\begin{array}{l}\text { Sachsen- } \\
\text { Anhalt }\end{array}$ & Stark II & 2010 & & $2012-2016$ & 0,513 \\
\hline $\begin{array}{l}\text { Schles- } \\
\text { wig- } \\
\text { Holstein }\end{array}$ & $\begin{array}{l}\text { Konsolidierungs- } \\
\text { hilfen }\end{array}$ & 2012 & 16 & $2012-2021^{1}$ & $\begin{array}{l}\text { 0,95 für Gesamt- } \\
\text { maßnahmen, 0,75 } \\
\text { für Entschul- } \\
\text { dungsfonds im } \\
\text { engeren Sinne }\end{array}$ \\
\hline
\end{tabular}

Anmerkung: Baden-Württemberg, Sachsen und Thüringen haben keine Programme, $1=$ Änderung bis 2018 geplant, 2 = der abgedeckten Kommunen 
Während in Rheinland-Pfalz die Finanzierung zu gleichen Teilen zwischen teilnehmenden Kommunen, kommunalem Finanzausgleich und Land organisiert ist, übernimmt in Nordrhein-Westfalen das Land rund $60 \%$ der Mittel des kommunalen Stärkungspaktes. In Hessen wiederum übernimmt das Land, wie in Sachsen-Anhalt, $100 \%$ der Kosten, während der Entschuldungsfonds in SchleswigHolstein vor allen Dingen über den kommunalen Finanzausgleich gespeist wird. Ein solcher Vergleich muss offen lassen, inwieweit der Anteil der Länder durch originäre Landesmittel oder indirekt über eine zurückhaltende Dotation des KFA finanziert wird.

In der Regel schließen die Bundesländer und die Kommunen einen Vertrag ab, in dem die Konsolidierungsmaßnahmen festgeschrieben werden. Im Gegenzug erhalten die Kommunen dafür die Finanzhilfen. Die Haushaltsüberwachung ist dabei wieder unterschiedlich geregelt. So muss in Nordrhein-Westfalen etwa jede beteiligte Kommune einen jährlichen Haushaltssanierungsplan vorlegen, aus dem der Defizitabbau hervorgehen soll. In Nordrhein-Westfalen, dem Land mit den Kommunen mit den höchsten Kassenkrediten, ist der sogenannte „Stärkungspakt Stadtfinanzen“ zweistufig organisiert. So gibt es eine Reihe Kommunen, die in der ersten Stufe verpflichtet wurden teilzunehmen (34 Städte), während auf einer zweiten Stufe rund 27 Kommunen freiwillig teilnehmen. Die Konsolidierungshilfen von Seiten der Länder bzw. der horizontalen kommunalen Ebene sind ein wesentlicher Baustein der Entlastung der besonders verschuldeten Kommunen. Insbesondere kann den Kommunen damit auch ein Weg aus der sogenannten „Vergeblichkeitsfalle“ gezeigt werden, das heißt, dass sich die Konsolidierungsanstrengungen auch lohnen. Der kommunale Haushalt muss mit dem Geld aus dem Stärkungspakt innerhalb von fünf Jahren (Stufe 1) bzw. sieben Jahren (Stufe 2) ausgeglichen sein. Im Kern handelt es sich jedoch um temporäre Hilfsmaßnahmen, d.h. mittel- und langfristig ist der Haushaltsausgleich von den Kommunen selbst zu leisten („Kommunalisierung des Lückenschlusses“).

Insgesamt können verschiedene Strategien der Entschuldungsprogramme ausgemacht werden (vgl. Tab. 8), die teilweise auch kombiniert werden (z.B. Hessen):

- Teilweise Übernahme auslaufender Kredite durch eine Landesinvestitionsbank (Hessen, Sachsen-Anhalt) oder das Land (Rheinland-Pfalz),

- Zins- und teilweise Tilgungshilfen (Brandenburg, Niedersachsen, Mecklenburg-Vorpommern) sowie

- Zuschüsse für den Gesamthaushalt (Nordrhein-Westfalen, Bayern). 
Tabelle 8: Eigenschaften der Stärkungspakte und Entschuldungsprogramme

\begin{tabular}{|c|c|c|c|c|}
\hline Land & Ziel & $\begin{array}{l}\text { Finanzierung } \\
\text { des Fonds }\end{array}$ & Voraussetzungen & $\begin{array}{l}\text { Rechtliche } \\
\text { Grundlage }\end{array}$ \\
\hline Bayern & $\begin{array}{l}\text { Haushaltskon- } \\
\text { solidierung, } \\
\text { Schuldentilgung }\end{array}$ & Land & $\begin{array}{l}\text { Stringente Auflagen: } \\
\text { muss zur Schuldentilgung } \\
\text { verwendet werden, Aus- } \\
\text { schöpfung aller eigenen } \\
\text { Einnahmemöglichkeiten, } \\
\text { Strukturschwäche }\end{array}$ & $\begin{array}{l}\text { Art. 11 FAG } \\
\text { (Finanzaus- } \\
\text { gleichsgesetz) }\end{array}$ \\
\hline $\begin{array}{l}\text { Branden- } \\
\text { burg }\end{array}$ & $\begin{array}{l}\text { Haushaltskon- } \\
\text { solidierung, } \\
\text { eigene Anstren- } \\
\text { gungen fördern }\end{array}$ & Land & $\begin{array}{l}\text { Erhebliche eigene An- } \\
\text { strengungen }\end{array}$ & $\begin{array}{l}\text { Brandenburgi- } \\
\text { sches Finanz- } \\
\text { ausgleichsge- } \\
\text { setz } 16 \\
\text { BbgFAG, } \\
\text { Abschnitt } 5\end{array}$ \\
\hline Hessen & $\begin{array}{l}\text { Tilgung: Land } \\
\text { übernimmt je } \\
46 \% \text { der Schul- } \\
\text { den der ärmsten } \\
\text { Kommunen, } \\
\text { Zinsverbilli- } \\
\text { gung }\end{array}$ & Land & $\begin{array}{l}\text { Auswahl der Kommunen } \\
\text { nach Kennzahlsystem }\end{array}$ & $\begin{array}{l}\text { Schutzschirm- } \\
\text { gesetz vom } \\
14.05 .2012\end{array}$ \\
\hline $\begin{array}{l}\text { Mecklen- } \\
\text { burg- } \\
\text { Vorpom- } \\
\text { mern }\end{array}$ & $\begin{array}{l}\text { Zinszuschüsse, } \\
\text { Ausgleich } \\
\text { unmittelbarer } \\
\text { Defizite }\end{array}$ & Land & $\begin{array}{l}\text { Beantragung, kein } \\
\text { Rechtsanspruch. Mittel- } \\
\text { fristiger Finanzplan darf } \\
\text { kein weiteres Defizit } \\
\text { ausweisen }\end{array}$ & $\begin{array}{l}\S 10 \text { Absatz } 1 \\
\text { FAG M-V }\end{array}$ \\
\hline $\begin{array}{l}\text { Nieder- } \\
\text { sachsen }\end{array}$ & $\begin{array}{l}\text { Zins- und Til- } \\
\text { gungshilfen }\end{array}$ & $\begin{array}{l}\text { Hälfte Lan- } \\
\text { desbeitrag, } \\
\text { Hälfte } \\
\text { Kommunaler } \\
\text { Finanzaus- } \\
\text { gleich } \\
\text { (KFA) }\end{array}$ & $\begin{array}{l}\text { Unterdurchschnittliche } \\
\text { Steuerkraft und über- } \\
\text { durchschnittliche Kas- } \\
\text { senkredite }\end{array}$ & $\S 14$ a-e NFAG \\
\hline $\begin{array}{l}\text { Nordrhein- } \\
\text { Westfalen }\end{array}$ & $\begin{array}{l}\text { Konsolidie- } \\
\text { rungshilfen }\end{array}$ & $\begin{array}{l}\text { 3,5 Mrd. } \\
\text { Euro Land, } \\
\text { 2,35 Mrd. } \\
\text { Euro KFA }\end{array}$ & $\begin{array}{l}\text { Klarer Sanierungskurs. } \\
\text { Besonders betroffene } \\
\text { Kommunen pflichtige } \\
\text { Teilnahme, auch freiwil- } \\
\text { lige Teilnahme möglich }\end{array}$ & $\begin{array}{l}\text { Stärkungspakt- } \\
\text { gesetz vom } \\
09.12 .2011\end{array}$ \\
\hline $\begin{array}{l}\text { Rheinland- } \\
\text { Pfalz }\end{array}$ & $\begin{array}{l}\text { Liquiditätskre- } \\
\text { dite tilgen, } \\
\text { Zinslasten } \\
\text { mindern }\end{array}$ & $\begin{array}{l}\text { Drittel Land, } \\
\text { Drittel KFA, } \\
\text { Drittel Ei- } \\
\text { genanteil } \\
\text { teilnehmen- } \\
\text { der Kommu- } \\
\text { nen }\end{array}$ & $\begin{array}{l}\text { Kommunen mit Kassenk- } \\
\text { rediten }\end{array}$ & $\S 17 \mathrm{~b}$ LFAG \\
\hline Saarland & $\begin{array}{l}\text { Konsolidie- } \\
\text { rungshilfen }\end{array}$ & Land & $\begin{array}{l}\text { Sanierungsauflagen der } \\
\text { Kommunalaufsichtsbe- }\end{array}$ & $\begin{array}{l}\text { Kommunaler } \\
\text { Entlastungs- }\end{array}$ \\
\hline
\end{tabular}




\begin{tabular}{|c|c|c|c|c|}
\hline Land & Ziel & $\begin{array}{l}\text { Finanzierung } \\
\text { des Fonds }\end{array}$ & Voraussetzungen & $\begin{array}{l}\text { Rechtliche } \\
\text { Grundlage }\end{array}$ \\
\hline & & & hörde & $\begin{array}{l}\text { fonds (KELF), } \\
2014 \text { kommt } \\
\text { noch gesonder- } \\
\text { tes Gesetz }\end{array}$ \\
\hline $\begin{array}{l}\text { Sachsen- } \\
\text { Anhalt }\end{array}$ & $\begin{array}{l}\text { Teilablösung } \\
\text { investiver } \\
\text { Schulden, Zin- } \\
\text { sentlastung }\end{array}$ & Land & $\begin{array}{l}\text { Beantragung von Kom- } \\
\text { munen mit fundierten } \\
\text { Krediten möglich }\end{array}$ & $\begin{array}{l}\text { STARKII - } \\
\S 18 \text { FAG }\end{array}$ \\
\hline $\begin{array}{l}\text { Schleswig- } \\
\text { Holstein }\end{array}$ & $\begin{array}{l}\text { Haushaltskon- } \\
\text { solidierung }\end{array}$ & $\begin{array}{l}0,15 \text { Mrd. } \\
\text { Euro Land, } \\
0,8 \text { Mrd. } \\
\text { Euro KFA }\end{array}$ & $\begin{array}{l}\text { Kommunen, die mind. } \\
\text { fünfmal den Haushalt- } \\
\text { sausgleich verfehlt haben }\end{array}$ & $\begin{array}{l}\text { §16a FAG SH, } \\
\text { Richtlinie über } \\
\text { die Gewährung } \\
\text { von Konsoli- } \\
\text { dierungshilfen }\end{array}$ \\
\hline
\end{tabular}

Anmerkung: Baden-Württemberg, Sachsen und Thüringen haben keine Programme

Eine weitere Möglichkeit der Länder, den Kommunen zu helfen, ist die bessere Ausgestaltung der kommunalen Finanzausgleichssysteme. Die Entwicklung der Finanzausgleichsmasse muss im besonderen Maße mit der Entwicklung der Sozial- und Jugendhilfeausgaben Schritt halten. Hinzu kommt, dass die Länder sich dem Konnexitätsprinzip „Wer bestellt, bezahlt“ nicht entledigen sollten, sondern zum Beispiel die zusätzlichen Kosten der Kommunen für den gemeinsamen Schulunterricht von behinderten und nichtbehinderten Kindern und Jugendlichen übernehmen. Die Bürgermeisterbefragung 2014 hat dies als den dringendsten Punkt aus Sicht der Bürgermeister identifiziert.

Aus Sicht der Länder sind überdies noch Kommunal- und Verwaltungsreformen ein Weg zur Haushaltskonsolidierung. In den vergangenen Jahrzehnten fanden zahlreiche Gemeindereformen statt, und es wurden - wie etwa in Niedersachsen die Ebene der Regierungsbezirke abgeschafft. ${ }^{30}$ Dennoch gibt es zwischen den Bundesländern eine große Varianz in den Einwohnerzahlen und der Zahl der Gemeinden. So gibt es in Nordrhein-Westfalen 396 Städte und Gemeinden, während es in dem viereinhalbmal kleineren Reinland-Pfalz 2.306 Gemeinden gibt. Die durchschnittliche Gemeindegröße beträgt daher 45.133 Einwohner (NRW) bzw. 1.736 Einwohner in Rheinland-Pfalz. Effizientere Gemeindestrukturen sind

30 Vgl. Bogumil, J. /Holtkamp, L.: Kommunalpolitik und Kommunalverwaltung - Eine policyorientierte Einführung, Wiesbaden, 2006. 
- gerade auch im Hinblick auf die Haushaltskonsolidierung - eine wichtige Maßnahme. $^{31}$

\section{Maßnahmen des Bundes}

In der Föderalismusreform I aus dem Jahre 2006 wurde die Abschaffung des Durchgriffs des Bundes auf die Kommunen nach Art. 84 (1) GG verabschiedet. Seitdem darf der Bund den Kommunen keine Aufgaben übertragen, insbesondere also solche nicht, die Kosten verursachen. Ein wichtiges Ziel der damaligen Reform war also die Stärkung des Konnexitätsprinzips. Mittlerweile dürfen Aufgaben an die Kommunen nur auf Basis der jeweiligen Landesgesetze abgegeben werden. Jede politische Ebene sollte demnach auch die Kosten der Aufgaben tragen, die sie im Gesetzgebungsprozess verabschiedet und diese nicht - auch nicht durch Mischfinanzierungen - auf andere Ebenen verlagern. ${ }^{32}$

Ein Beispiel hierfür ist der Ausbau der Kinderbetreuungsmaßnahmen. Diese vom Bund veranlasste Politik sah vor, jedem Kind einen Kinderbetreuungsplatz ab 2013 zu garantieren (Kinderförderungsgesetz aus dem Jahre 2008). Ein zentraler Bestandteil war ab dem 1. August 2013, nach Abschluss der Ausbauphase, ein Rechtsanspruch auf einen Betreuungsplatz für alle Kinder vom vollendeten ersten bis zum vollendeten dritten Lebensjahr. Die Finanzierung des Ausbaus wurde jedoch nicht alleine vom Bund getragen: Von den insgesamt 12 Milliarden Euro, die für den Ausbau benötigt wurden, trug der Bund mit 4 Milliarden Euro rund ein Drittel. Ab 2014 beteiligt sich der Bund dauerhaft mit jährlich 770 Millionen an den laufenden Kosten, die jedoch deutlich höher liegen. Jenseits der Wünschbarkeit einer solchen Politik bleibt der Fakt, dass die Kommunen einen Großteil der Kosten dieses Bundesgesetzes trugen.

Ein weiteres Beispiel ist die Grundsicherung im Alter und bei Erwerbsminderung. Dies ist eine Leistung der Sozialhilfe (SGB XII § 41) und ist im Prinzip ein Äquivalent zur Sozialhilfe - nur für einen ausgewählten Personenkreis. Auch hier ist die gesetzliche Grundlage ein Bundesgesetz. Die Kosten fielen in der Vergangenheit bei den Kommunen an. Durch den Kompromiss in der Gemeindefinanzkommission übernimmt der Bund ab 2014 die Grundsicherung im Alter, immerhin rund 5,1 Milliarden Euro für 2015.

31 Vgl. Junkernheinrich, M.: Neuordnung von Gemeinden: Drei Schritte auf dem Weg zur Sicherung leistungsfähiger Verbandsgemeinden in Rheinland-Pfalz, in: Junkernheinrich, M./Lorig, W. (Hg.): Kommunalreformen in Deutschland, Baden-Baden 2013, 245-268.

32 Allerdings ist sicher zu stellen, dass insbesondere bei den Sozial- und Jugendhilfeausgaben, die einen höheren kommunalen Durchführungsspielraum aufweisen, hinreichende Anreize zur wirtschaftlichen Aufgabenerfüllung der örtlichen Träger erhalten bleiben müssen. 
Wie die statistischen Analysen gezeigt haben, sind gerade der Anteil der Sozialhilfeempfänger (SGB II-Quote) und die Arbeitslosenquote mit die wichtigsten Einflussgrößen für die kommunalen Kassenkredite. Würde der Bund weiter den Weg zu einer höheren und teilweise vollständigen Kostenübernahme solcher Soziallasten gehen - etwa durch eine Nettoentlastung bei der Eingliederungshilfe für Behinderte oder die Übernahme der Kosten der Unterkunft -, wäre ein wichtiger Schritt zur ursachenadäquaten Entlastung der Kommunen getan.

\section{Zusammenfassung}

Die empirischen Ergebnisse haben gezeigt, dass sowohl exogene als auch endogene Faktoren die Verschuldung treiben. Zwar sind exogene Faktoren gewichtiger, dennoch erhöhen politische Faktoren die Erklärungskraft der Determinanten der Kassenkredite beträchtlich. Bemerkenswert sind auch die unterschiedlichen Befunde für die Analysen über die Zeit sowie den Querschnittsregressionen. Bei einer zeitraumbasierten Analyse spielen die endogenen politischen Faktoren eine weitaus größere Rolle und sind statistisch signifikant, im Gegensatz zur Analyse zu einem Zeitpunkt.

Eine Problemlösung erfordert ein abgestimmtes Verhalten aller relevanten Akteure, also insbesondere von Kommunen, Ländern und Bund. Hinsichtlich des kommunalen Beitrags ergab die Auswertung der Bürgermeisterbefragung 2014 neben der Einnahmenerhöhung bei der Grund- und Gewerbesteuer auch verschiedene ausgabenseitige Maßnahmen. Insgesamt forderten die Bürgermeister eine bessere finanzielle Ausstattung sowie eine Stärkung des Konnexitätsprinzips, d.h. es sollten weniger Aufgaben an die Kommunen delegiert werden, ohne gleichzeitig diese Aufgaben finanziell zu kompensieren. Die Erfahrungen mit den Konsolidierungsanstrengungen auf kommunaler Ebene in einigen Bundesländern zeigen ein ähnliches Bild. In der Regel wird zur Konsolidierung ein vielfältiger Mix aus Einnahmenverbesserungen und Ausgabensenkungen angewendet. Institutionelle Änderungen werden etwa im Bereich der Budgetierung vorgenommen (Doppik). Verfahren wie Benchmarking und kommunale Leistungsvergleiche spielen eine untergeordnete Rolle. Die Implementation eines Frühwarnsystems für die kommunale Ebene, ähnlich wie beim Stabilitätsrat, wäre wünschenswert.

Die Länder haben in den vergangenen Jahren auf die in einigen Bundesländern ansteigende Kommunalverschuldung mit Entschuldungsprogrammen und „Stärkungspakten" reagiert. In immerhin 10 von 13 Flächenländern gibt es solche Programme. Diese unterscheiden sich jedoch in Umfang, Finanzierungsstruktur 
und Zahl der teilnehmenden Kommunen. Auch die Instrumente differieren erheblich. So findet mitunter eine teilweise Übernahme von auslaufenden Krediten statt oder es werden Zins- und Tilgungshilfen gewährt. Nordrhein-Westfalen geht am weitesten und gewährt temporäre Zuschüsse für den Haushaltsausgleich. Eine weitere Möglichkeit zur Verbesserung der finanziellen Situation sind Reformen des Gebietszuschnitts. Die Zahl und die durchschnittlichen Gemeindegrößen unterscheiden sich deutlich zwischen den Bundesländern. Kommunale Zusammenlegungen und Fusionen wären daher wünschenswert.

Auch der Bund hat in den vergangenen Jahren die Kommunen entlastet. So war die Entscheidung in der Gemeindefinanzkommission zur vollständigen Übernahme der Kosten für die Grundsicherung im Alter eine wichtige Entlastung für die Kommunen. Dieses Beispiel verdeutlicht einen möglichen Weg, nämlich die weitere Übernahme von kommunalen Ausgaben, die durch Leistungsgesetze auf Bundesebene veranlasst werden. Gleichzeitig würde dies eine Stärkung des Konnexitätsprinzips bedeuten und gerade den sozioökonomisch höher belasteten Kommunen ursachenadäquat helfen. 